Please do not remove this page

RMIT

UNIVERSITY

\title{
Differential Work-Function Enabled Bifunctional Switching in Strontium Titanate Flexible Resistive Memories
}

Rahman, Md. Ataur; Abbas, Sherif; Ahmed, Taimur; Spencer, Michelle; Walia, Sumeet; Sriram, Sharath; Bhaskaran, Madhu

https://researchrepository.rmit.edu.au/esploro/outputs/9921861402701341/filesAndLinks?institution=61RMIT_INST\&index=null

Rahman, M. A., Abbas, S., Ahmed, T., Spencer, M., Walia, S., Sriram, S., \& Bhaskaran, M. (2020). Differential Work-Function Enabled Bifunctional Switching in Strontium Titanate Flexible Resistive Memories. ACS Applied Materials and Interfaces, 12(6), 7326-7333. https://doi.org/10.1021/acsami.9b20585 Document Version: Accepted Manuscript

Published Version: https://doi.org/10.1021/acsami.9b20585

Repository homepage: https://researchrepository.rmit.edu.au

(c) 2020 American Chemical Society

Downloaded On 2023/04/26 19:56:18 +1000 
Thank you for downloading this document from the RMIT Research Repository.

The RMIT Research Repository is an open access database showcasing the research outputs of RMIT University researchers.

RMIT Research Repository: http://researchbank.rmit.edu.au/

\section{Citation:}

Rahman, M, Abbas, S, Ahmed, T, Spencer, M, Walia, S, Sriram, S and Bhaskaran, M 2020, 'Differential work-function enabled bifunctional switching in strontium titanate flexible resistive memories', ACS Applied Materials and Interfaces, vol. 12, no. 6, pp. 7326-7333.

See this record in the RMIT Research Repository at:

https://researchbank.rmit.edu.au/view/rmit:57486

Version: Accepted Manuscript

Copyright Statement:

(C) 2020 American Chemical Society

\section{Link to Published Version:}

https://dx.doi.org/10.1021/acsami.9b20585 


\title{
Differential work-function enabled bifunctional switching in strontium titanate flexible resistive memories
}

\author{
Md. Ataur Rahman, ${ }^{\mathrm{a} *}$ Sherif Abdulkader Tawfik, ${ }^{\mathrm{b}}$ Taimur Ahmed, ${ }^{\mathrm{a}}$ Michelle J.S. Spencer, ${ }^{\mathrm{b}}$ \\ Sumeet Walia, ${ }^{a}$ Sharath Sriram, ${ }^{a}$ and Madhu Bhaskaran ${ }^{\mathrm{a}_{*}}$ \\ ${ }^{a}$ Functional Materials and Microsystems Research Group and the Micro Nano Research \\ Facility, RMIT University, Melbourne, Victoria 3001, Australia \\ ${ }^{\mathrm{b}}$ School of Science, RMIT University, GPO Box 2476 Melbourne, Victoria 3001 Australia \\ *Corresponding authors email: mdataur.rahman@ student.rmit.edu.au, \\ madhu.bhaskaran@rmit.edu.au
}

\section{Keywords}

resistive switching memory, bipolar switching, complementary switching, work-function difference, flexibility

\begin{abstract}
Multifunctional electronic memories capable of demonstrating both analog and digital switching on-demand are extremely attractive for miniaturization of electronics without significant drain on energy consumption. Simultaneously translating functionality onto mechanically conformable platforms will further enhance their suitability. Here, we demonstrate the ability to engineer multi-functionality in strontium titanate (STO) based resistive random-access memories (ReRAM) on a flexible polyimide (PI) platform. By utilising different bottom electrodes of various work functions while the top electrode is fixed, differential work-functions are induced in STO, to induce bipolar (BP) or complementary (CS) switching behaviours whenever required. This work-functions difference induced bifunctional switching on flexible platform reveals a streamlined route for achieving flexible artificial neural networks, high density integration, and logic operation using a single ReRAM.
\end{abstract}




\section{Introduction}

Future non-volatile memories will encompass miniaturized data storage, low power operation, low switching current and high endurance/retention performance. ${ }^{1-2}$ Resistive random-access memories (ReRAM) with an active metal oxide switching layer sandwiched in a metal-insulator-metal (MIM) stack have drawn considerable attention due to their highdensity integration and superior memory performance. ${ }^{3-5}$ Depending on the applications, the behaviour of ReRAMs can be classified into two types: analog bipolar switching and digital threshold switching.

The analog ReRAM is particularly important because of its amenability to mimic synaptic behaviour for neuromorphic computing, where resistance changes gradually for the SET and RESET process. ${ }^{6-7}$ So, the analog ReRAM is a fundamental building block for an artificial neural network. On the other hand, digital ReRAM devices play a crucial role to control the unintended leakage current due to parasitic paths around neighbouring cells during high density integration. ${ }^{8}$ In addition, due to the abrupt state change, and stable resistance state, digital ReRAM is the foundation for logic operations. ${ }^{9}$ Therefore, it is of great interest to investigate if analog bipolar switching and digital complementary switching can be realised in a single metal oxide - this will create a single platform for artificial neural networks, high density integration, and logic operations.

When two dissimilar metals with different work functions are electrically isolated, an arbitrary potential difference may exist between them known as Volta potential difference. ${ }^{10-}$

${ }^{12}$ It is reported that this intrinsic potential applies an electric field to soft breakdown the thin insulating layer in the MIM stack. ${ }^{12-13}$ Herein, we report the effect of the work-function difference on the switching behaviour of thin $\mathrm{SrTiO}_{3-x}$ (STO) based MIM stack. The work functions of common inert materials such as $\mathrm{Pt}, \mathrm{Pd}$, and $\mathrm{Au}$ and hence the work-function differences of Pt-vacuum-Ti, Pd-vacuum-Ti, and Au-vacuum-Ti are estimated using density functional theory (DFT). Ti is chosen as its work function is much lower than that of $\mathrm{Pt}, \mathrm{Pd}$, and $\mathrm{Au}$ and it also works as a reservoir for the oxygen vacancy induced switching process for the STO-based device. ${ }^{14}$

In order to observe the influence of work-function difference, a room temperature deposited oxygen-deficient $\mathrm{SrTiO}_{3-x}$ (STO) based MIM stack on a flexible polyimide (PI) platform has been fabricated. STO is selected as the functional oxide due to its ability to harbour oxygen vacancies $\left(\mathrm{V}_{\mathrm{O}}^{\bullet \bullet}\right)$ whose concentration can be controlled. ${ }^{15-16} \mathrm{PI}$ is chosen as the substrate due 
to its suitability for use in flexible and wearable applications, with architectures for optimized skin-electrode contact. ${ }^{17}$ It is hypothesised that the replacement of higher work function based $\mathrm{Pt}$ or $\mathrm{Pd}$ metal in bottom electrode $\mathrm{Pt} / \mathrm{Ti} / \mathrm{STO} / \mathrm{Pt} / \mathrm{Ti}$ or $\mathrm{Pd} / \mathrm{Ti} / \mathrm{STO} / \mathrm{Pd} / \mathrm{Ti}$ stack with a lower work function Au will influence the switching behaviour to change from analog bipolar to digital complementary switching behaviour. The fabricated devices are characterised with the two different functional switching mechanisms explained.

\section{Results and Discussions}

\section{Estimation of work-function difference and device fabrication}

When a thin metal oxide is sandwiched between two metals of different work functions, the intrinsic potential difference results in the creation of an electric field across the thin oxide layer causing a soft breakdown of the insulation between the top and bottom electrode. ${ }^{13}$ In MIM structure of resistive switching memory, the metals are isolated by an insulating metal oxide layer. We calculated the work functions of these metals and hence the work-function differences when the metals are isolated by vacuum using density functional theory (DFT) in an aim to understand its effect on the switching behaviours of our devices. In this work, we estimate the work-function differences for the Ti-vacuum-Pt, $\mathrm{Ti}-\mathrm{vacuum}-\mathrm{Pd}$, and $\mathrm{Ti}-$ vacuum-Au interfaces by calculating the work function for the isolated surfaces, and then following the procedure in Ref. ${ }^{18}$ Using density functional theory, the calculated work functions for Pt (111), $\mathrm{Pd}$ (111), $\mathrm{Au}$ (111), and Ti (001) surfaces are $5.74 \mathrm{eV}, 5.41 \mathrm{eV}$, $5.19 \mathrm{eV}$, and $4.13 \mathrm{eV}$, respectively. Figure 1 displays the plane-averaged potential of the four structures, along with the values of work function for each surface. Details of these calculations are provided in the Supporting Information. 

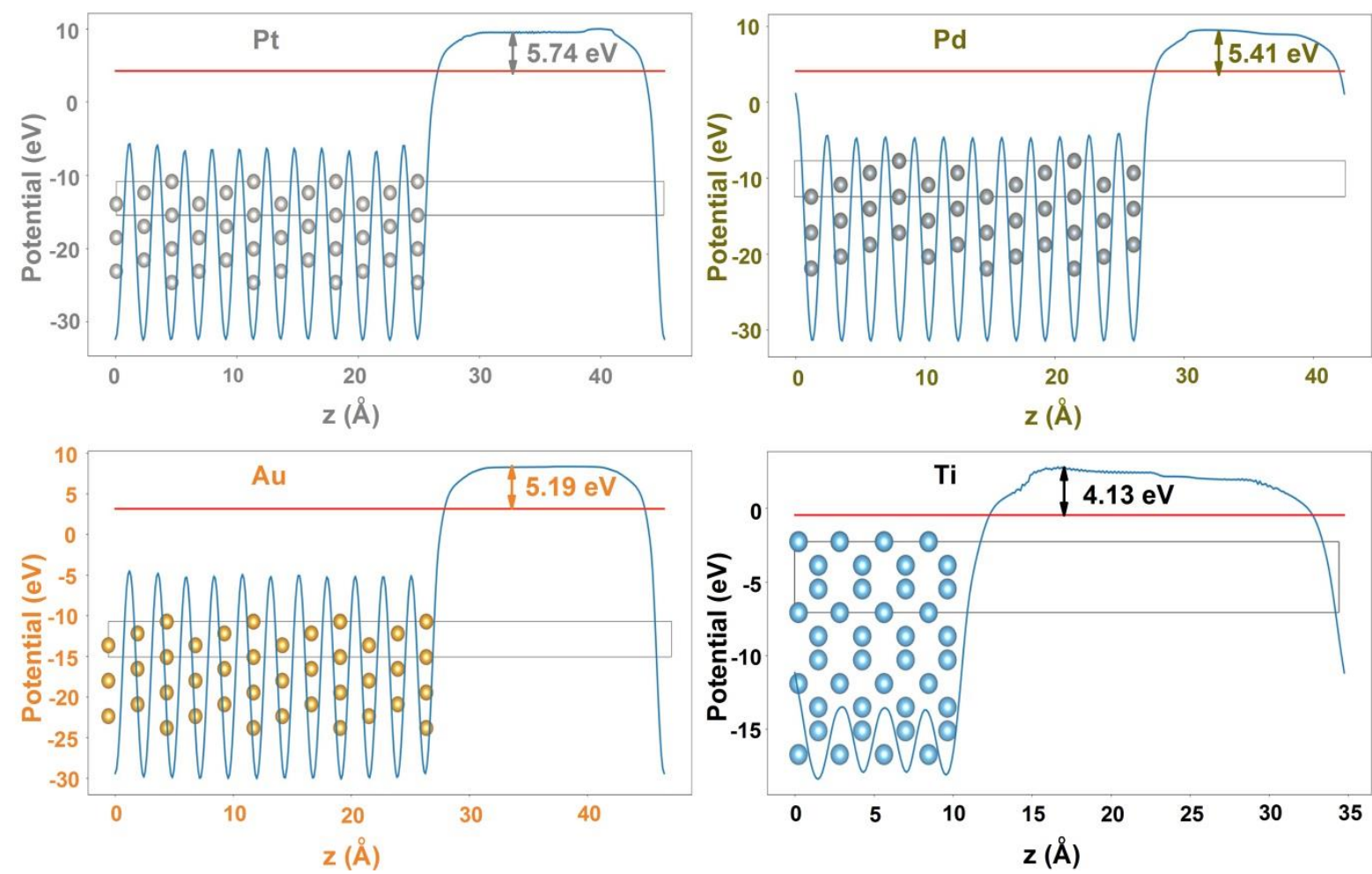

Figure 1. The plane-averaged potential of the four surfaces, namely Pt, Pd, Au, and Ti. The Fermi level is depicted as a solid red horizontal line, and the work function values are displayed.

The potential differences due to the work-function differences for the $\mathrm{Pt}, \mathrm{Pd}$, and $\mathrm{Au}$ surfaces with respect to the Ti electrode are calculated via the equation $\delta \psi_{\mathrm{Ti}}=\left(\phi_{S}-\phi_{\mathrm{Ti}}\right) / e$, where $\phi_{S}$, is the work function for the $\mathrm{Pt}, \mathrm{Pd}$, or Au surface, and $\phi_{\mathrm{Ti}}$ is the work function of the Ti surface. The work function differences between the reference $\mathrm{Ti}$ and $\mathrm{Pt}, \mathrm{Pd}$ and $\mathrm{Au}$ are 1.61, 1.28 and $1.06 \mathrm{eV}$ respectively. The difference in the work functions between the Ti surface and the $\mathrm{Pt}, \mathrm{Pd}$, or $\mathrm{Au}$ surface results in flow of electric charge from the Ti surface (lower work function) towards $\mathrm{Pt}, \mathrm{Pd}$, and $\mathrm{Au}$ surfaces (higher work functions). This results in the creation of a net positive potential on the Ti surface, and a net negative potential on the Pt, $\mathrm{Pd}$, and $\mathrm{Au}$ surface. It is to be noted that the equilibrium half-cell potential of metal/STO interface can change the calculated work-function difference in vacuum; we believe that this will not change the order of the work-function difference of the three surfaces as the half-cell potential is also applied to the reference material (Ti). A schematic diagram for the workfunction difference calculation for the three interfaces is presented in Figure 2. 

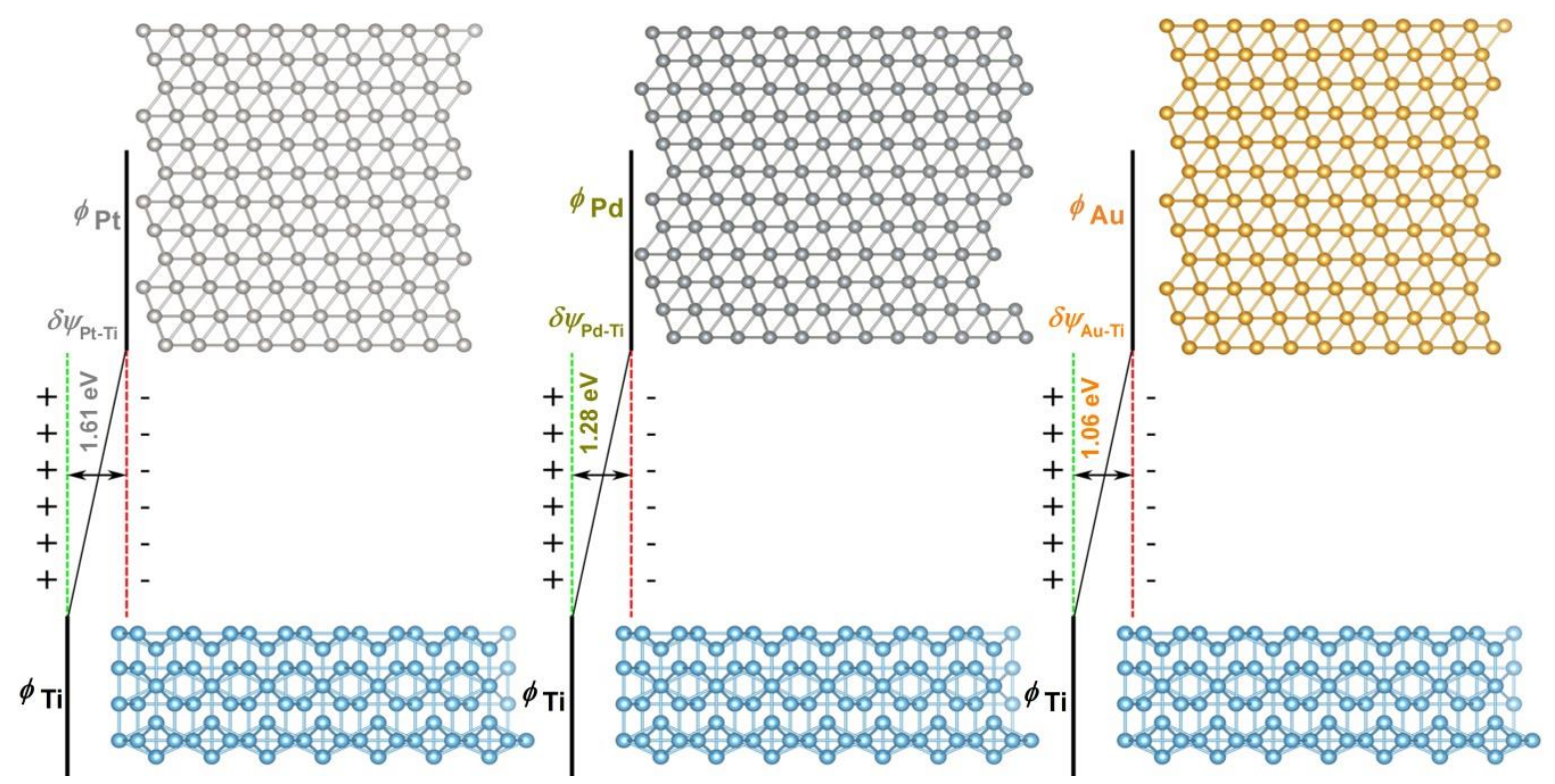

Figure 2. A schematic diagram for the $\mathrm{Pt} / \mathrm{Ti}, \mathrm{Pd} / \mathrm{Ti}$, and $\mathrm{Au} / \mathrm{Ti}$ interfaces, showing the potential difference exists due to the work function differences.

In order to comprehend the effect of the work-function difference on the switching behaviour of STO based memory, three different MIM stacks on flexible polyimide (PI) substrate are fabricated as illustrated in Figure 3, along with its schematic representation. Devices are fabricated as per the following configuration: Pt/Ti/STO/Pt/Ti/PI, Pd/Ti/STO/Pd/Ti/PI, and $\mathrm{Au} / \mathrm{Ti} / \mathrm{STO} / \mathrm{Au} / \mathrm{Cr} / \mathrm{PI}$; henceforth referred to as STO/Pt/PI, STO/Pd/PI, and STO/Au/PI devices, respectively. We hypothesize that though the calculated work-function difference in vacuum may be influenced by the STO/metal interaction, this will also apply to the reference material (Ti), and hence change the reference potential for the three materials. Therefore, even though the specific work function values of the Au, Pd and Pt might be slightly affected by the presence of STO, we believe that this will not change the order of the work-function difference of the three stacks. Bottom Ti or $\mathrm{Cr}$ is used for the adhesion of $\mathrm{Pt}, \mathrm{Pd}$, or $\mathrm{Au}$ with the PI substrate while top $\mathrm{Ti}$ is used as the oxygen reservoir as well as adhesion of top $\mathrm{Pt}, \mathrm{Pd}$, or $\mathrm{Au}$. The bottom Pt, Pd, or Au were selected for their variations in work-function difference with respect to Ti. Moreover, the formation free energy of the metal electrode oxide plays a vital role in determining the device behaviours. The larger the formation free energy of oxide, the smaller the resistance of that device with that electrode. The active electrode Ti has a very large formation free energy of oxide; hence it can easily form $\mathrm{Ti}$ oxide which works as an oxygen reservoir for our devices. On the other hand, cathodic electrodes Pt, Pd or Au has very low free energy of formation of oxides. As a result they don't have any stable oxides. The impact of the counter electrode for ECM and VCM type devices are reported elaborately 
in the literatures. ${ }^{19-21}$ The top Pt, Pd, or Au serve as an inert material to passivate Ti, thereby preventing the formation of $\mathrm{TiO}_{2}$ due to exposure to environmental oxygen. ${ }^{14}$ The STO thin films have been characterised in our previous works. ${ }^{22-26}$ The oxygen vacancies created during the controlled deposition are mainly responsible for the resistive switching characteristics. $^{22-26}$

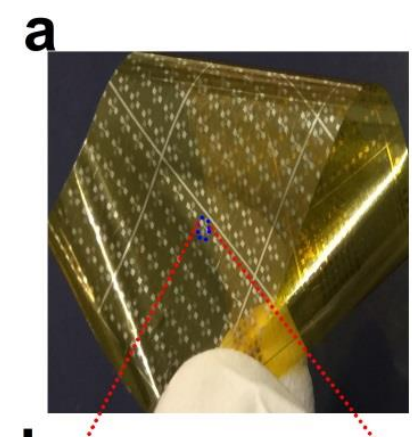

b.

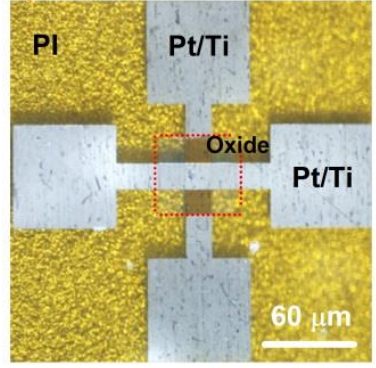

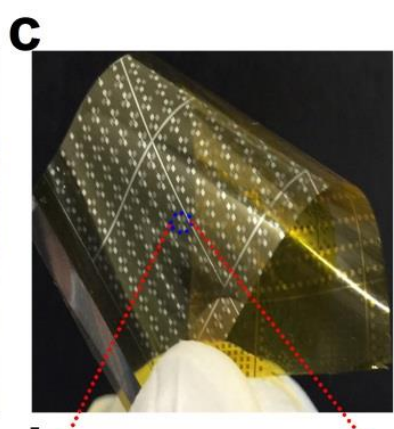

di

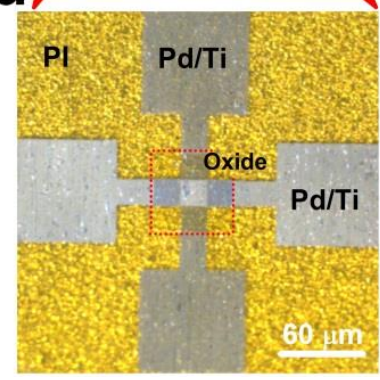

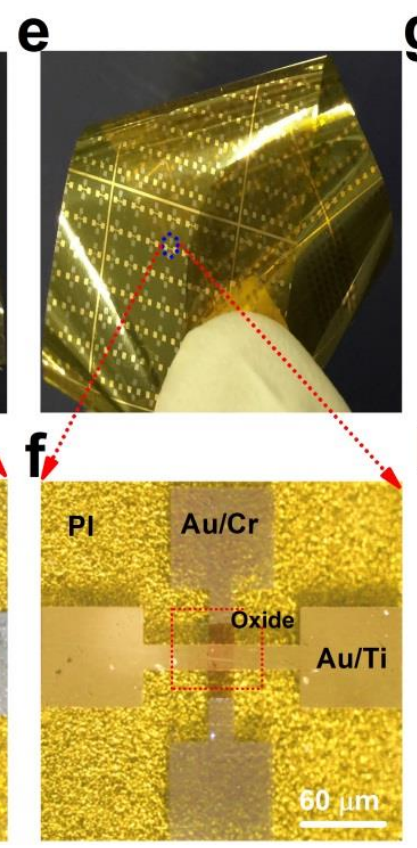

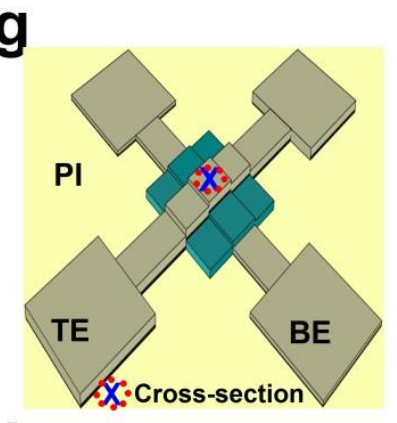

h

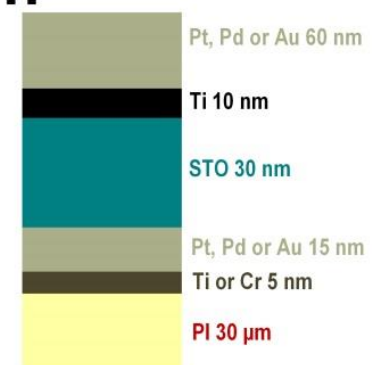

Figure 3. Fabricated devices on polyimide (PI) substrates. (a) Photograph and (b) optical micrograph of STO/Pt/PI, (c) photograph and (d) optical micrograph of STO/Pd/PI devices, and (e) photograph and (f) optical micrograph of $S T O / A u / P I$ devices. In all cases, devices range in size from $2 \times 2 \mu^{2}$ to $100 \times 100 \mu \mathrm{m}^{2}$ in (a, c, and e) with a $60 \times 60 \mu \mathrm{m}^{2}$ cross-point device shown in (b, d, and f). Depiction of device structure as (g) three-dimensional schematic and (h) cross-sectional representation with layer thicknesses.

\section{Electrical characterisation of STO based devices}

In order to activate the resistive switching behaviour in STO/Pt/PI, STO/Pd/PI, and STO/Au/PI devices, DC voltage sweeps of varying polarity are applied to the bottom electrode (Schottky contact, STO/Pt) of the devices while the top electrode (ohmic contact, Ti/STO) is grounded. ${ }^{14}$ Both the STO/Pt/PI and STO/Pd/PI devices exhibit bipolar (BP) switching while the STO/Au/PI device shows complementary switching (CS) as depicted in Figure 4. The size dependent switching behaviours of the stacks $\left(40 \times 40 \mu \mathrm{m}^{2}, 20 \times 20 \mu \mathrm{m}^{2}\right.$, $10 \times 10 \mu \mathrm{m}^{2}$ ) are also presented in Figure S1. It is well established that nanofilament is the mainly responsible for switching behaviour of STO based devices; hence no considerable 
switching variation is seen for different sizes. The higher the work-function difference, the more easily it is for the thin STO layer to attain soft breakdown due to applied voltage, ${ }^{13}$ and hence the required switching voltages for STO/Pt/PI, STO/Pd/PI, and STO/Au/PI are -0.85 to $+1.15 \mathrm{~V},-1.1$ to $+1.2 \mathrm{~V}$, and -1.9 to $+1.9 \mathrm{~V}$, respectively. This can be further extending down if the OFF current behaviours of the three devices are carefully observed. The intrinsic potential which is reported as the nanobattery, ${ }^{27}$ exactly exemplifies the OFF current characteristics of the memristor. The nanobattery results in a deviation of zero crossing property of $I-V$ characteristics of ideal memristors to a non-zero crossing behaviour. Figures 4d, e, \&f illustrate the zoomed in versions of linear behaviour as shown in the inset. The OFF state current and ON state current are labelled as A and B, respectively. The magnitude of the OFF state currents at $0 \mathrm{~V}$ are $3.1 \mathrm{nA}, 1.4 \mathrm{nA}$ and $0.5 \mathrm{nA}$ for STO/Pt/PI, $S T O / P d / P I$, and STO/Au/PI devices, respectively. The higher the intrinsic work-function difference, the higher the OFF state current consequence lower switching voltage to easily soft breakdown the insulating oxide layer. It can be clearly seen in Figure 4 that the STO/Pd/PI shows lower switching ratio compared to that of the STO/Pt/PI device. It can be interpreted that higher the $\delta \psi$, the higher the switching ratio for the devices with bipolar switching behaviour.

a

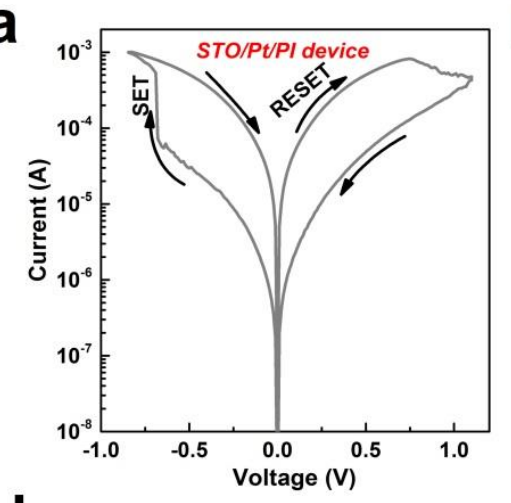

d

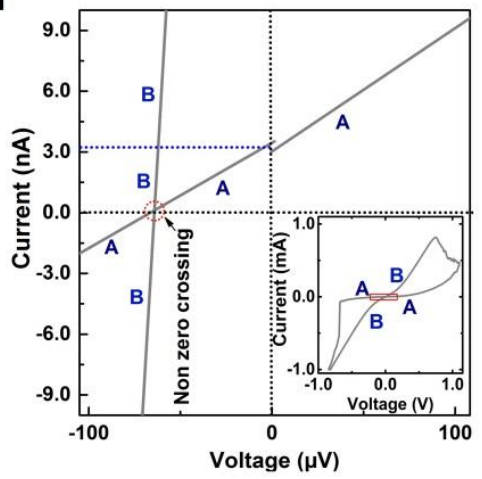

b

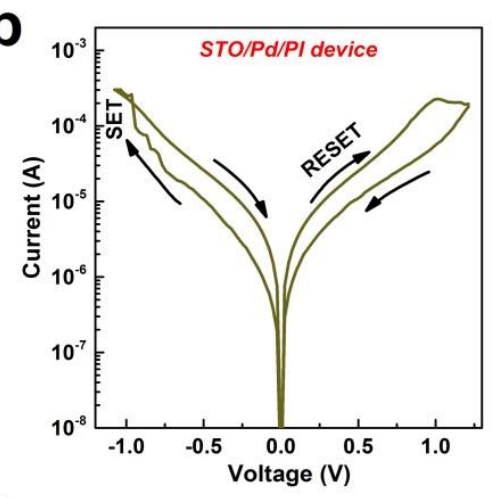

e

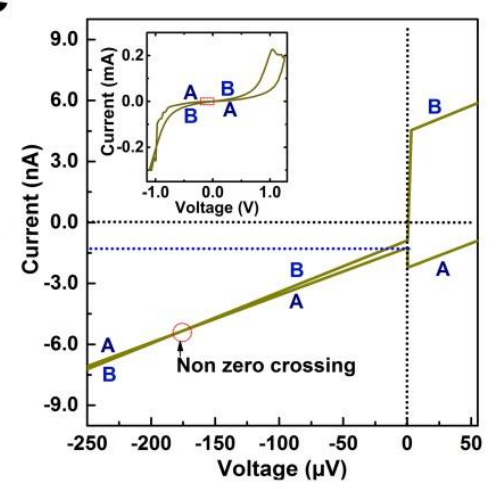

C

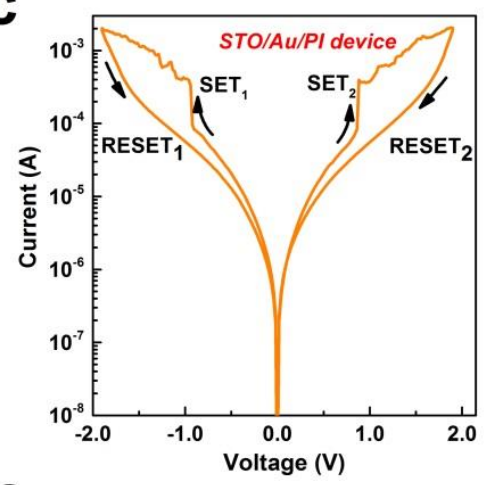

f

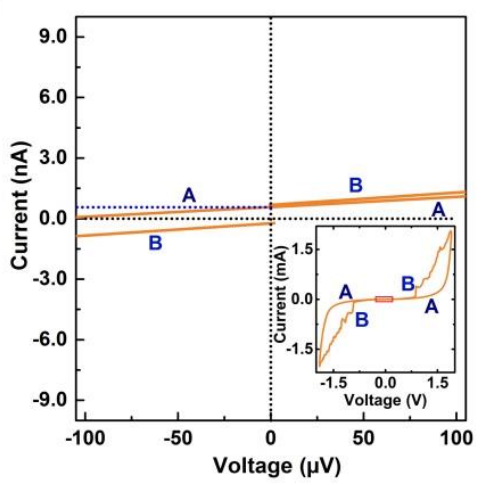


Figure 4. Influence of work-function difference. (a) Polarity-dependent bipolar switching behaviour of STO/Pt/PI device where the work-function difference of $\mathrm{Pt}$ and $\mathrm{Ti}$ is $1.61 \mathrm{eV}$. (b) Polaritydependent bipolar switching behaviour of STO/Pd/PI device due to the difference of work-function of $\mathrm{Pd}$ and $\mathrm{Ti}$ is $1.28 \mathrm{eV}$. (c) CS memory behaviour of STO/Au/PI device where the work-function difference of $\mathrm{Au}$ and $\mathrm{Ti}$ is $1.06 \mathrm{eV}$. (d) OFF current of STO/Pt/PI; inset shows linear switching. (e) OFF current of STO/Pd/PI device; inset shows the linear bipolar switching. (f) OFF current of $S T O / A u / P I$ device where inset depicts the linear complementary switching.

The absorbed moisture could have a significant influence on resistive switching behaviour. The oxidation of oxygen ions and reduction of moisture at the anodic and cathodic interface, respectively play a vital role to initiate the switching process as repoted. ${ }^{28-30}$ The gas bubbles created during the oxidation-reduction process can cause the structural damage for symmetric cell as reported by the K.Liu et al. ${ }^{30}$ To see any effect of moisture content in our devices, we tested our STO/Pt/PI devices under various relative humidity levels from $10 \%$ to $90 \%$. The experimental set up is depicted in Figure S2. Figure 5a illustrates the high resistive, low resistive state and hence the switching ratio under various $\mathrm{Rh}$ conditions. The results affirm that there is no significant role of moisture on our device. From the HRTEM image, which we reported in our previous work revealed the existence of $\mathrm{Ti}_{2} \mathrm{O}_{3}$ layer seamlessly stick to the Ti TE indicating the absence of gas eruption from the anodic surface. ${ }^{31}$ Hence we believe that due to the absence of oxygen bubbles at the TE region, no reaction takes place from the absorbed moisture in the BE. This observation is further supported by the recent study for the asymmetric device. ${ }^{30}$ Hence, the absence of moisture effect reaffirm the switching variation of our devices is due to the work-function difference. Furthermore, the statistical analysis has been carried out for 10 devices of each kind. We observed BP switching behaviour for $\mathrm{STO} / \mathrm{Pt} / \mathrm{PI}$ and STO/Pd/PI devices and CS behaviour for STO/Au/PI devices. The results reaffirm that lower work-function difference require higher switching voltages and vice versa as seen from Figure 5b,c, \&d. Though there is a deviation of switching voltages as seen from the Figure $5 b \& c$, the trend of switching voltages with respect to the work-function differences are not changed. However, slight variation of switching voltage is expected from cell to cell. It may be because of the Volta potential of a metal is affected by external fields, slightly by temperature change, varies with crystal face exposed to metal oxide and is altered by adsorbed atoms and the surface force fields. ${ }^{10}$ Figure $5 \mathrm{~d}$ describes the statistical data for high resistive state and low resistive state. It is evident from Figure $5 \mathrm{~d}$ that there is a large deviation of $\mathrm{HRS}$ for $\mathrm{STO} / \mathrm{Pd} / \mathrm{PI}$ devices inferring that $\mathrm{Pt}$ and $\mathrm{Au}$ are the suitable $\mathrm{BE}$ materials for the BP and TS switching behaviour for stability. As a result, we only discussed 
STO/Pt/PI and STO/Au/PI devices as the representative BP and CS behaviours correspondingly in the following section.

a

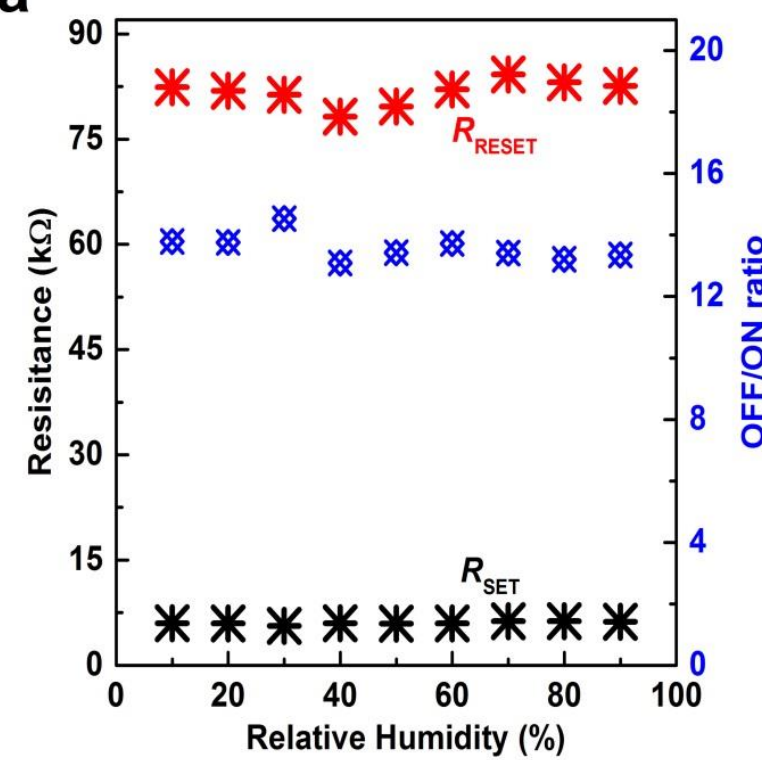

C

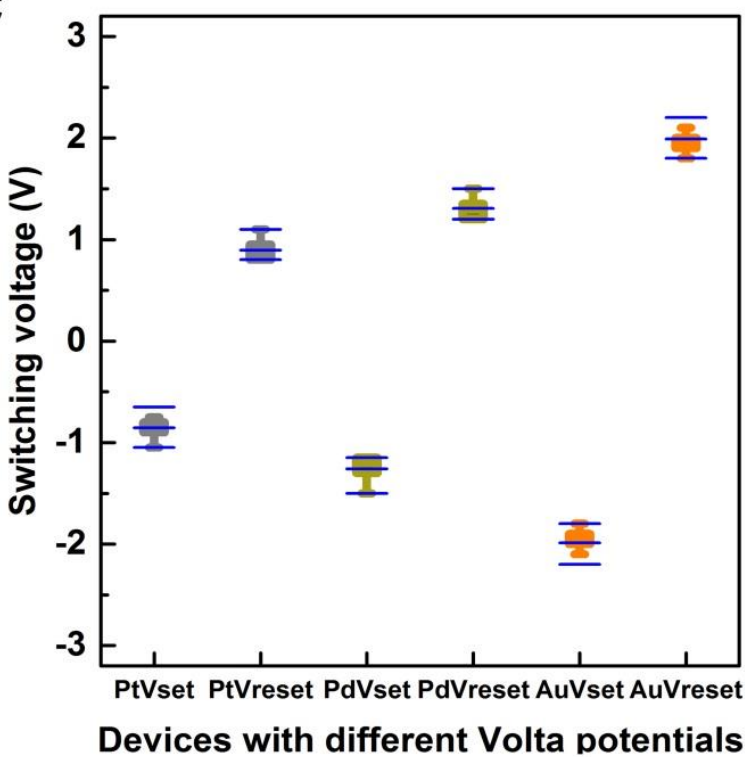

b

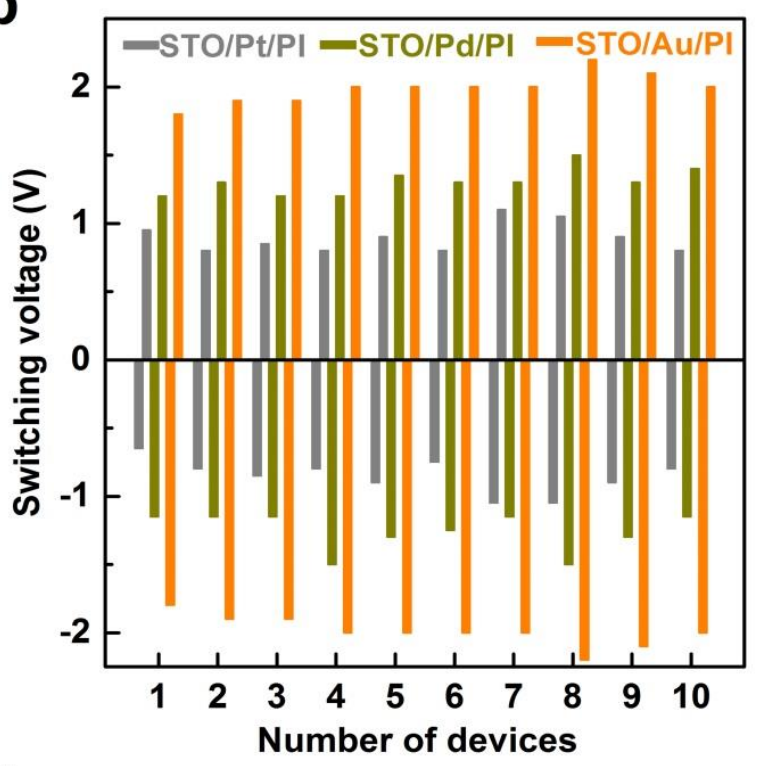

d

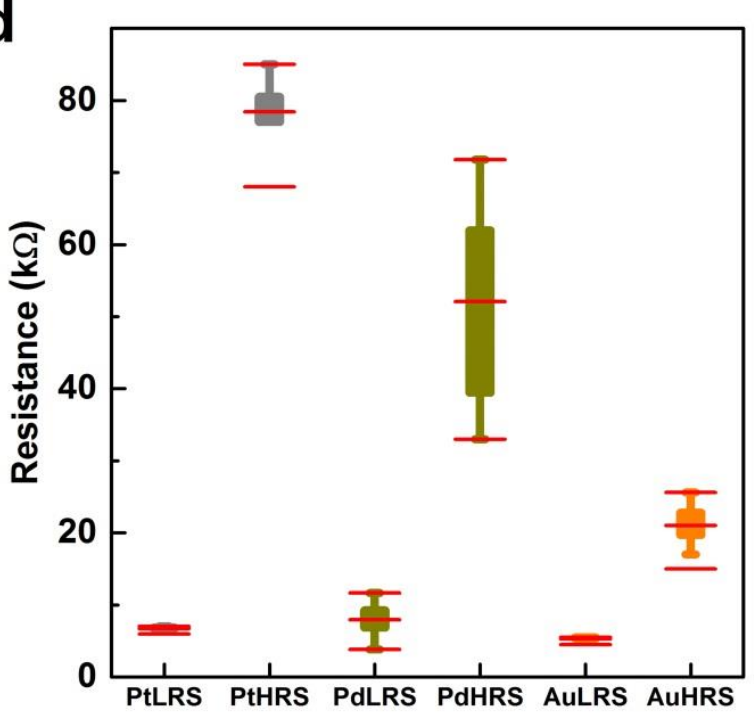

Devices with different Volta potentials

Figure 5. Effect of moisture and statistical analysis. a) HRS, LRS and switching ratio with respect to the relative humidity variation. b) Variation of switching voltages for 10 devices of each kind of STO/Pt/PI, STO/Pd/PI, and STO/Au/PI. c) Deviation and mean value of set and reset voltages for Pt, Pd, and Au based devices. d) Deviation and mean value of LRS and HRS for Pt, Pd, and Au based devices. 


\section{Switching mechanisms and reason for differential switching}

Figure 6 illustrates the possible resistive switching mechanism based on the electrical and material characterization of our BP and CS devices. The inherent oxygen vacancies $\left(\mathrm{V}_{\mathrm{O}}^{\bullet \bullet}\right)$ in the $\mathrm{Ti}-\mathrm{O}^{6}$ octahedra that are created by preferentially removing the oxygen atoms due to heavy $\mathrm{Ar}^{+}$ion bombardment during the sputtering process are mainly responsible for the switching process of our MIM devices. ${ }^{24}$ It is well known that electroforming creates a localized channel for the current flow through STO. ${ }^{31}$ Moreover, the subsequent resistive switching is attributed to the alternate growth and rupture of this conductive pathway, through drift of oxygen vacancies $\mathrm{V}_{\mathrm{O}} \cdot \boldsymbol{\bullet}$ and Joule heating, under the influence of applied bias. ${ }^{32}$ Lower current compliance of $1 \mu \mathrm{A}$ during the electroforming, is insufficient for the driving bias to migrate $\mathrm{V}_{\mathrm{O}}^{\bullet \bullet}$ for complete formation of the conductive pathway. A compliance current of $50 \mu \mathrm{A}$ generates higher density of $\mathrm{V}_{\mathrm{O}}$ sufficient for the complete formation of conductive filaments at very low driving force. Under the influence of negative bias to the bottom electrode, positively charged $\mathrm{V}_{\mathrm{O}}^{\bullet}$ accumulate at STO/Pt interface whereas their density extends to the top electrode forming an extended oxygen deficient filamentary pathway; hence, LRS is achieved. Nevertheless, the application of positive bias to the bottom electrode repulses the positively charged oxygen vacancies resulting in a discontinuous filamentary pathway which switches the device to the HRS. The electrical behaviours and corresponding schematics are represented in Figure 6a. It is noteworthy that to observe bipolar switching, some type of symmetry breaking is necessary such as asymmetric lower applied bias $(-0.85 \mathrm{~V}$ to $+1.15 \mathrm{~V}$ ), electrode structure (using an active Ti electrode as the anode and inert $\mathrm{Pt}$ electrode as the cathode) or an asymmetric switching layer stack. ${ }^{33}$ Due to the larger Pt workfunction, the polarity of potential due to work-function difference is such that it reinforces the negative applied voltage and opposes the positive applied voltage to the Pt electrode with respect to the Ti electrode. Hence the $V_{\mathrm{SET}}(-0.85 \mathrm{~V})$ is comparatively inferior to the $V_{\mathrm{RESET}}$ $(+1.15 \mathrm{~V})$ resulting asymmetric behaviour. The stable BP switching for 50 cycles, retention for $10^{3} \mathrm{~s}$ and endurance for $10^{3}$ cycles without any degradation are discussed in Supporting Information and the related figures are illustrated in Supporting Information Figure S3 indicate the stable bipolar switching behaviour of our STO/Pt/PI devices.

In contrast to higher resistance of the virgin STO/Pt/PI devices, the STO/Au/PI devices demonstrate forming-free behaviour as shown in Figure $5 \mathrm{~b}$. Migration of the $\mathrm{V}_{\mathrm{O}}^{\bullet \bullet}$ in the STO 
thin film fluctuates due to the tensile strain of $\mathrm{Au}$ on the film. ${ }^{34}$ It has been predicted in another report that a higher tensile strain results in a more effective migration of the $\mathrm{V}_{\mathrm{O}}^{\bullet \bullet}$ in STO.${ }^{35}$ Hence, we hypothesize that the tensile strain of $\mathrm{Au} 4.4 \%,{ }^{36}$ which is twice the strain of Pt $2.2 \%,{ }^{37}$ enhances of migration of $\mathrm{V}_{\mathrm{O}}^{\bullet}$ in the functional layer rendering the devices forming-free in nature.

A comment is required at this point regarding the forming-free behaviour of Au based device. Despite the migration of $\mathrm{V}_{\mathrm{O}}^{\bullet}$ increase due to the biaxial strain of $\mathrm{Au}$, the cell will still be in the OFF state with respect to the subsequent threshold switching. As a result, switching between the ON and OFF state can be accomplished by applying a voltage of opposite polarity and sufficiently high magnitude to the cell. ${ }^{38}$ The CS behaviour occurs due to the alternate formation and disruption of conductive filament in the surrounding area of the STO/Au and Ti/STO interfaces owing to the polarity of biasing voltage. ${ }^{9}$ The growth of the filament at an interface results in local low resistive state (LLRS) and rupture of the filament leads to local high resistive state (LHRS). As the intrinsic work-function difference is lower for $\mathrm{Au}-\mathrm{Ti}$ compared to $\mathrm{Pt}-\mathrm{Ti}$, a larger applied bias is needed to soft breakdown the thin STO layer in bottom electrode. ${ }^{13}$ Consequently, sudden increase in current is observed which is reported as one of the common phenomena for CRS behaviour. Due to the requirement of higher RESET (RESET 1 and RESET $)_{2}$ voltage as the SET $\left(\mathrm{SET}_{1}\right.$ and $\left.\mathrm{SET}_{2}\right)$ voltage is superior than that of Pt-based devices, the four states $\mathrm{SET}_{1}, \mathrm{RESET}_{1}, \mathrm{SET}_{2}$ and RESET 2 are accessible to confirm the CS behaviour using a single metal oxide layer. The switching mechanism of the CS behaviour of STO based devices with an Au bottom electrode is elaborately discussed in our previous work. ${ }^{22}$

In brief, an increment of applied negative bias at the STO/Au interface causes a rapid surge in current, setting the device to LRS ( $\left.\mathrm{SET}_{1}\right)$, where a complete filamentary formation takes place between the top and bottom electrodes. ${ }^{8}$ The LRS occurs at $-1.0 \mathrm{~V}$ as shown in the Figure $6 \mathrm{~b}$. The applied negative voltage attracts the positively charged $\mathrm{V}_{\mathrm{O}}^{\bullet \bullet}$ creating a larger concentration towards the bottom STO/Au interface. A higher negative voltage results in higher accumulation of $\mathrm{V}_{\mathrm{O}}^{\bullet}$ at the STO/Au interface pushing the interface in a LLRS. Consequently, the shortfall of $\mathrm{V}_{\mathrm{O}}^{\bullet}$ at the Ti/STO interface causes LHRS. The combination of LLRS at STO/Au interface and LHRS at Ti/STO results a mixed RESET $_{1}$ (LLRS/LHRS) state. On the other hand, during the positive sweep to the bottom electrode, the $\mathrm{V}_{\mathrm{O}}$ are pushed towards the top Ti/STO interface which forms an intermediate LRS $\left(\mathrm{SET}_{2}\right)$ state, and further increment of positive voltage switches the device to a RESET 2 (LHRS/LLRS) state. Unlike 
the STO/Pt/PI asymmetric BP behaviour, STO/Au/PI based CS requires certain types of symmetry. It can be clearly seen from the CS behaviour that similar magnitude of $1.9 \mathrm{~V}$ is needed to switch the device in positive and negative direction. Figure $6 \mathrm{~b}$ depicts the schematic of the switching mechanism and corresponding electrical behaviour. The stable CS switching for 50 cycles, retention for $10^{3} \mathrm{~s}$ and endurance for $10^{3}$ cycles without any degradation are discussed elaborately in Supporting Information and the associated figures are presented in Figure S4 indicate the stable complementary switching behaviour of our $\mathrm{STO} / \mathrm{Pt} / \mathrm{PI}$ devices.

To further validate the effect of work-function difference, we replace the top Au and Pt used as inert electrodes by $\mathrm{Pd}$. The $\mathrm{Pd} / \mathrm{Ti} / \mathrm{STO} / \mathrm{Pt} / \mathrm{Ti} / \mathrm{PI}$ exhibits $\mathrm{BP}$ and $\mathrm{Pd} / \mathrm{Ti} / \mathrm{STO} / \mathrm{Au} / \mathrm{Cr} / \mathrm{PI}$ shows CS as shown in Supporting Information Figure S5. These results confirm that there is no contribution from the top layer in the switching process. It is also to be noted that the effect of interchanging Schottky and ohmic contact is reported in our previous work for Pt based electrode. ${ }^{23,31}$ From the report we can infer that due to the interchanging, switching direction might be varied but the switching types: BP switching for STO/Pt/PI and CS for STO/Au/PI would not be changed.

a

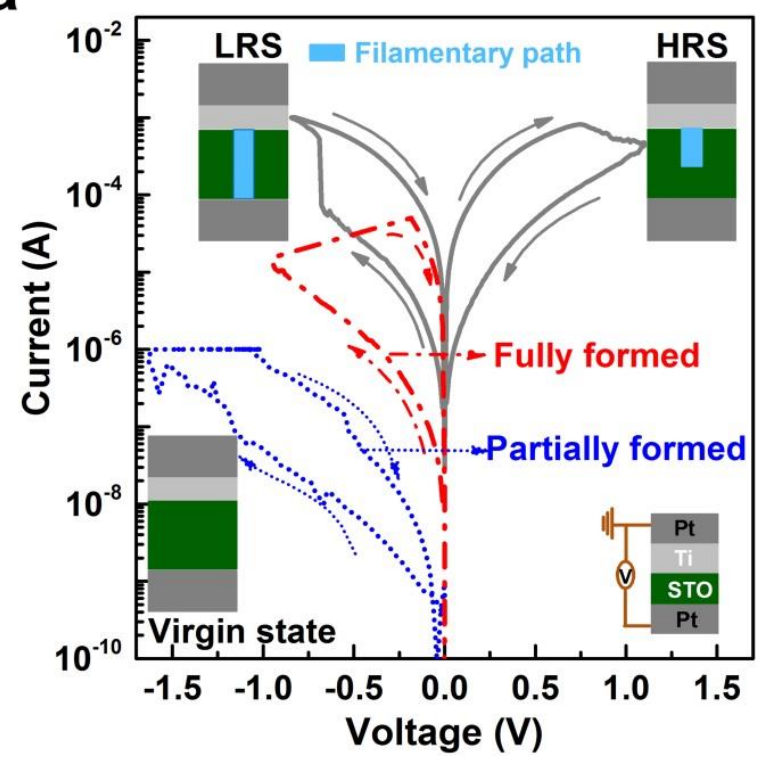

b

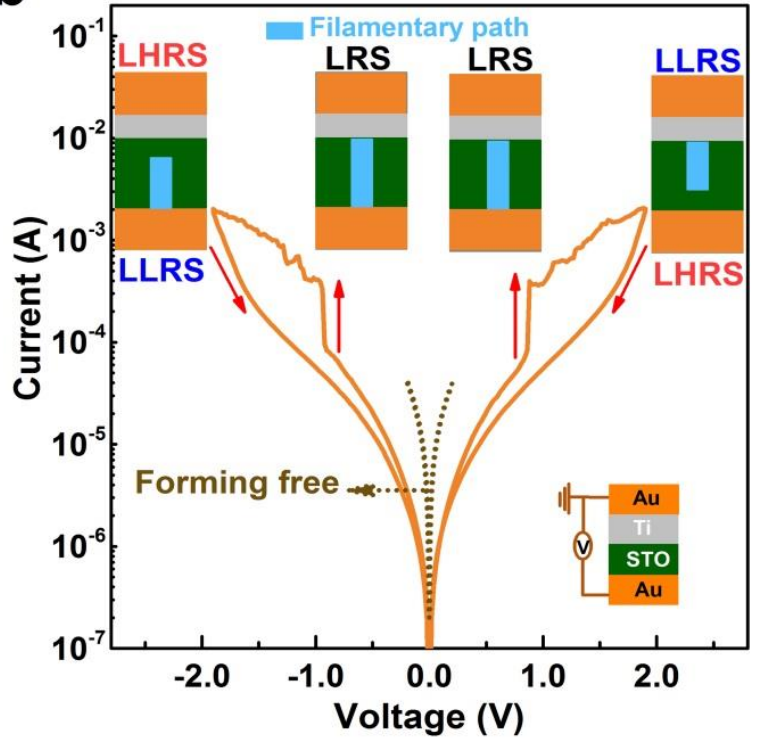

Figure 6. Schematic and electrical representation for bipolar and complementary switching mechanism. (a) The virgin state with no filamentary path as represented by green colour. The device is partially formed at low compliance current (CC) of $1 \mu \mathrm{A}$ and fully formed at $50 \mu \mathrm{A}$ with the application of negative bias at bottom Pt electrode. The negative voltage attracts all the positive $\mathrm{V}_{\mathrm{O}}$ towards the Pt/STO interface and extended till to the top Ti/STO interface resulting in LRS. The 
positive bias repels the $\mathrm{V}_{\mathrm{O}} \bullet$ and HRS is achieved. The HRS is represented by discontinues filamentary line. (b) The applied negative bias to the bottom Au electrode of forming-free device, switch the device to LRS state followed by LHRS at ohmic (Ti/STO) interface and LLRS at bottom electrode. Further increment of the bias, a depletion region is created to the top electrode as more positively charged oxygen vacancies moves toward the bottom electrode due to negative supply. Nevertheless, the positive bias drives the device to LRS before switching it LLRS at the top electrode and LHRS at the bottom electrode.

\section{Switching characteristics for curvilinear surfaces}

We fabricated STO-based devices on PI to demonstrate the feasibility of fabrication of flexible electronics. The resistive switching properties of the devices by affixing samples to a cylinder of $7 \mathrm{~mm}$ diameter are represented in Figure 7. Figure $7 \mathrm{~b}$ depicts the stable BP and CS of STO/Pt/PI and STO/Au/PI for 50 cycles, respectively. Figure 7c demonstrates the stable retention characteristics for $10^{4} \mathrm{~s}$ for BP and CS at a constant read voltage $\left(V_{\mathrm{READ}}\right)$ of $75 \mathrm{mV}$ and $1.2 \mathrm{~V}$, respectively. 

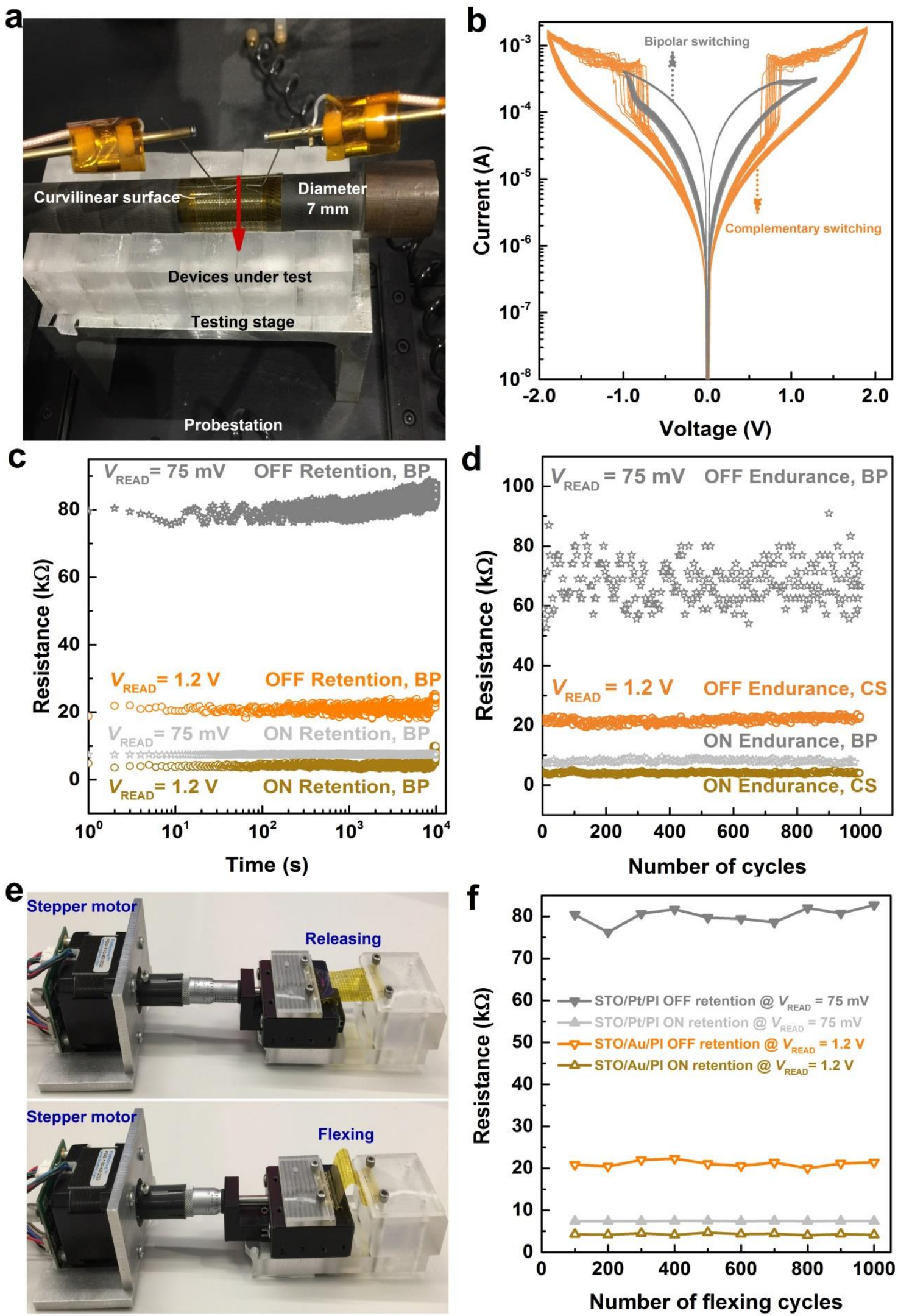

Figure 7. Evaluation of memory performance under curvature. (a) Measurement arrangement to induce tensile curvilinear strain through $7 \mathrm{~mm}$ diameter curvature during electrical characterisation. (b) $\mathrm{BP}$ and $\mathrm{CS}$ of $\mathrm{Pt} / \mathrm{Ti} / \mathrm{STO} / \mathrm{Pt} / \mathrm{Ti} / \mathrm{PI}$ and $\mathrm{Au} / \mathrm{Ti} / \mathrm{STO} / \mathrm{Au} / \mathrm{Cr} / \mathrm{PI}$ devices, respectively, for 50 cycles 
under curvature. (c) Retention tests for $10^{4} \mathrm{~s}$ under curvature. (d) Endurance over 1,000 cycles under curvature. (e) Testing set up for repeated bending cycles. (f) ON and OFF retention for 1,000 flexing cycles.

It is apparent from the retention test that $\mathrm{OFF} / \mathrm{ON}$ ratio is very low for both the $\mathrm{BP}$ and $\mathrm{CS}$ switching. However, the BP devices with such switching ratios are still applicable as synaptic device with excellent gradual switching as reported ${ }^{39}$ and CS devices with such switching ratios are reported for logic operation to implement the crossbar adder. ${ }^{9}$ The switching ratios can be improved by depositing STO in oxygen environment and exploiting thicker STO films. ${ }^{23}$ The endurance measurement for both cases is carried out using a train of pulses with duration of $1 \mathrm{~ms}$ as presented in Supporting Information Figure S3c and S4c.

A slight difference was observed in the switching ratio as shown in Figures S3, S4 but overall, there was not much change in electrical properties upon bending state. Most importantly, no degradation is observed after 50 switching cycles, $10^{4} \mathrm{~s}$ of retention and $10^{3}$ endurance cycles indicating good electrical reliability as well as mechanical stability under bending condition. We carried out further mechanical repeatability test under extreme bending condition. The stepper motor (Trinamic PD-110-42, Germany) which is run by a LabVIEW program to drive the micrometer screw as shown in the Figure $7 \mathrm{e}$ is used for bending and relaxing devices. It is to be noted that the device of interest is kept on the maximum bending point of the film. It can be clearly seen from Figure $7 f$ that there is no noticeable variation in the switching ratio for both STO/Pt/PI and STO/Au/PI devices for $10^{3}$ flexing-releasing cycles infering our device is mechanically stable to be potentially applicable for flexible electronics.

\section{Conclusion}

In conclusion, we have demonstrated bipolar switching and complementary switching behaviours in room temperature deposited oxygen-deficient STO thin films fabricated on flexible polyimide substrate. The resistive memories have exhibited stable non-volatile memory switching of 50 cycles, strong data retention time over $10^{3} \mathrm{~s}$ and cycling endurance of $10^{3}$ cycles indicating longer operational period of our MIM devices. It is demonstrated that the symmetric applied bias and lower work-function difference between top and bottom electrodes play a vital role in inducing complementary switching behaviour from asymmetric bipolar switching. Higher work-function difference on STO causes bipolar switching, while lower work-function difference creates complementary switching. Based on this insight, 
choosing appropriate electrodes at the interfaces will determine the switching behaviour. This allows a single material platform or memory element network to be programmed to be analog or digital, enabling neural networks and logic operations on demand. While there are opposing theories on the operating principle of VCM cells, the effect of potential due to work-function difference on STO based MIM devices is a major step in understanding the bifunctional switching behaviour.

\section{Experimental Details}

\section{Material deposition and device fabrication}

The MIM stack was fabricated on a $30 \mu \mathrm{m}$ PI substrate which was the chosen flexible substrate. The substrate was RCA cleaned and exposed to plasma cleaning in Ar environment for $60 \mathrm{~s}$. The bottom electrode comprised of $\mathrm{Cr}$ or $\mathrm{Ti}(5 \mathrm{~nm})$ and $\mathrm{Au}, \mathrm{Pt}$, or Pd (15 nm) were fabricated after pattering with $5214 \mathrm{E}$ photoresist, electron beam deposition at $0.3 \AA / \mathrm{s}$, and by standard lift-off procedures. The insulator layer consists of functional strontium titanate $(30 \mathrm{~nm})$ that was deposited from a commercial STO target from Testbourne Ltd. with a purity of $99.95 \%$ after patterning using the photolithography process. To create the oxygen-deficient STO, the deposition was conducted in a $100 \% \mathrm{Ar}$ atmosphere and at a pressure of $3.5 \times 10^{-3}$ Torr at room temperature. At such low pressure, heavy $\mathrm{Ar}+$ ion bombardment during deposition preferentially removes the oxygen atoms which results sub-stoichiometric

$\mathrm{SrTiO}_{3-x}{ }^{23-24}$ The deposition performed at room temperature facilitates the realization of devices on flexible platform which cannot withstand high temperatures. Subsequently, standard lift-off was carried out to achieve the features as patterned. Similar steps were followed for top electrode as bottom electrode except the thicknesses which are $\mathrm{Ti}(10 \mathrm{~nm})$ and Au or Pt or Pd (60 nm).

\section{Electrical characterisation}

An Agilent B2912 A source/measure unit was used to acquire the current-voltage behaviours of work-function difference enabled bi-functional switching of STO based memories. 


\section{AUTHOR INFORMATION}

\section{Corresponding authors:}

*E-mails: mdataur.rahman@student.rmit.edu.au and madhu.bhaskaran@rmit.edu.au

\section{Author Contributions}

The manuscript was written through contributions of all authors, and all authors have given approval to the final version of the manuscript.

\section{Notes}

The authors declare no competing financial interest.

\section{ACKNOWLEDGMENTS}

We acknowledge personnel and project funding from the Australian Research Council through DE160100023. This work was performed in part at the Micro Nano Research Facility at RMIT University in the Victorian Node of the Australian National Fabrication Facility (ANFF).

\section{SUPPORTING INFORMATION}

The Supporting Information is available free of charge on the ACS Publications website at DOI:

Details of the Volta Potentials Calculations, Effect of device size on the switching behaviour, Test set up for moisture effect, Bipolar Switching Behaviour of STO/Pt/PI, Complementary switching behaviour of STO/Au/PI, and switching behaviour of $\mathrm{Pd} / \mathrm{Ti} / \mathrm{STO} / \mathrm{Pt} / \mathrm{Ti}$ and $\mathrm{Pd} / \mathrm{Ti} / \mathrm{STO} / \mathrm{Au} / \mathrm{Ti}$. 


\section{References}

1. Qian, K.; Tay, R. Y.; Nguyen, V. C.; Wang, J.; Cai, G.; Chen, T.; Teo, E. H. T.; Lee, P. S., Hexagonal Boron Nitride Thin Film for Flexible Resistive Memory Applications. Advanced Functional Materials 2016, 26 (13), 2176-2184.

2. Rahman, F.; Ahmed, T.; Walia, S.; Mayes, E.; Sriram, S.; Bhaskaran, M.; Balendhran, S., Reversible Resistive Switching Behaviour in CVD Grown, Large Area $\mathrm{MoO}_{\mathrm{x}}$. Nanoscale 2018, 10 (42), 19711-19719.

3. Kang, B. S.; Ahn, S.-E.; Lee, M.-J.; Stefanovich, G.; Kim, K. H.; Xianyu, W. X.; Lee, C. B.; Park, Y.; Baek, I. G.; Park, B. H., High-Current-Density CuOx/InZnOxThin-Film Diodes for Cross-Point Memory Applications. Advanced Materials 2008, 20 (16), 3066-3069. 4. $\quad$ Lee, M.-J.; Kim, S. I.; Lee, C. B.; Yin, H.; Ahn, S.-E.; Kang, B. S.; Kim, K. H.; Park, J. C.; Kim, C. J.; Song, I.; Kim, S. W.; Stefanovich, G.; Lee, J. H.; Chung, S. J.; Kim, Y. H.; Park, Y., Low-Temperature-Grown Transition Metal Oxide Based Storage Materials and Oxide Transistors for High-Density Non-volatile Memory. Advanced Functional Materials 2009, 19 (10), 1587-1593.

5. $\quad$ Kügeler, C.; Meier, M.; Rosezin, R.; Gilles, S.; Waser, R., High Density 3D Memory Architecture Based on the Resistive Switching Effect. Solid-State Electronics 2009, 53 (12), 1287-1292.

6. $\quad$ Kim, S.; Kim, H.; Hwang, S.; Kim, M. H.; Chang, Y. F.; Park, B. G., Analog Synaptic Behavior of a Silicon Nitride Memristor. ACS Appl Mater Interfaces 2017, 9 (46), 40420-40427.

7. Choi, S.-J.; Kim, G.-B.; Lee, K.; Kim, K.-H.; Yang, W.-Y.; Cho, S.; Bae, H.-J.; Seo, D.-S.; Kim, S.-I.; Lee, K.-J., Synaptic Behaviors of a Single Metal-Oxide-Metal Resistive Device. Applied Physics A 2011, 102 (4), 1019-1025.

8. $\quad$ Linn, E.; Rosezin, R.; Kugeler, C.; Waser, R., Complementary Resistive Switches For Passive Nanocrossbar Memories. Nat Mater 2010, 9 (5), 403-406.

9. $\quad$ Breuer, T.; Siemon, A.; Linn, E.; Menzel, S.; Waser, R.; Rana, V., A HfO -Based $^{2}$ Complementary Switching Crossbar Adder. Advanced Electronic Materials 2015, 1 (10), 1500138.

10. Loeb, L. B., The Contact Potential Difference or Volta Potential. In Static Electrification, Loeb, L. B., Ed. Springer Berlin Heidelberg: Berlin, Heidelberg, 1958; pp 3258.

11. Adamson, A. W., Physical Chemistry of Surfaces. 6th ed.; Wiley: New York, 1997. 12. Zhang, H. Z.; Ang, D. S.; Zhou, Y.; Wang, X. P., Enlarged Read Window in the Asymmetric ITO/HfO $/$ TIN Complementary Resistive Switch. Applied Physics Letters 2017, 111 (4), 043501.

13. Hadi, M. S.; Sugii, N.; Wakabayashi, H.; Tsutsui, K.; Iwai, H.; Kakushima, K., Resistive Switching Properties of a Thin $\mathrm{SiO}_{2}$ Layer with $\mathrm{CeO}_{x}$ Buffer Layer on $\mathrm{n}+$ and $\mathrm{p}+\mathrm{Si}$ Bottom Electrodes. Microelectronics Reliability 2016, 63, 42-45.

14. Waser, R.; Dittmann, R.; Staikov, G.; Szot, K., Redox-Based Resistive Switching Memories - Nanoionic Mechanisms, Prospects, and Challenges. Advanced Materials 2009, 21 (25-26), 2632-2663.

15. Koehl, A.; Wasmund, H.; Herpers, A.; Guttmann, P.; Werner, S.; Henzler, K.; Du, H.; Mayer, J.; Waser, R.; Dittmann, R., Evidence For Multifilamentary Valence Changes in Resistive Switching $\mathrm{SrTiO}_{3}$ Devices Detected by Transmission X-Ray Microscopy. APL Materials 2013, 042102,1-8.

16. Muenstermann, R.; Menke, T.; Dittmann, R.; Waser, R., Coexistence of Filamentary and Homogeneous Resistive Switching in Fe-Doped $\mathrm{SrTiO}_{3}$ Thin-Film Memristive Devices. Adv Mater 2010, 22 (43), 4819-4822. 
17. Liu, Y.; Pharr, M.; Salvatore, G. A., Lab-on-Skin: A Review of Flexible and Stretchable Electronics for Wearable Health Monitoring. ACS Nano 2017, 11 (10), 96149635.

18. Jin, Y.; Liu, M.; Zhang, C.; Leygraf, C.; Wen, L.; Pan, J., First-Principle Calculation of Volta Potential of Intermetallic Particles in Aluminum Alloys and Practical Implications. Journal of The Electrochemical Society 2017, 164 (9), C465-C473.

19. Dietz, G. W.; Antpöhler, W.; Klee, M.; Waser, R., Electrode Influence on the Charge Transport Through $\mathrm{SrTiO}_{3}$ Thin Films. Journal of Applied Physics 1995, 78 (10), 6113-6121. 20. Yang, J. J.; Strachan, J. P.; Miao, F.; Zhang, M.-X.; Pickett, M. D.; Yi, W.; Ohlberg, D. A. A.; Medeiros-Ribeiro, G.; Williams, R. S., Metal/TiO ${ }_{2}$ Interfaces for Memristive Switches. Applied Physics A 2011, 102 (4), 785-789.

21. Tappertzhofen, S.; Waser, R.; Valov, I., Impact of Tte Counter-Electrode Material on Redox Processes In Resistive Switching Memories. ChemElectroChem 2014, 1 (8), 12871292.

22. Rahman, M. A.; Ahmed, T.; Walia, S.; Sriram, S.; Bhaskaran, M., Oxygen-Deficient Strontium Titanate Based Stretchable Resistive Memories. Applied Materials Today 2018, $13,126-134$.

23. Nili, H.; Walia, S.; Balendhran, S.; Strukov, D. B.; Bhaskaran, M.; Sriram, S., Nanoscale Resistive Switching in Amorphous Perovskite Oxide (a-SrTiO 3 ) Memristors. Advanced Functional Materials 2014, 24 (43), 6741-6750.

24. Nili, H.; Walia, S.; Kandjani, A. E.; Ramanathan, R.; Gutruf, P.; Ahmed, T.; Balendhran, S.; Bansal, V.; Strukov, D. B.; Kavehei, O.; Bhaskaran, M.; Sriram, S., DonorInduced Performance Tuning of Amorphous $\mathrm{SrTiO}_{3}$ Memristive Nanodevices: Multistate Resistive Switching and Mechanical Tunability. Advanced Functional Materials 2015, 25 (21), 3172-3182.

25. Nili, H.; Ahmed, T.; Walia, S.; Ramanathan, R.; Kandjani, A. E.; Rubanov, S.; Kim, J.; Kavehei, O.; Bansal, V.; Bhaskaran, M.; Sriram, S., Microstructure and Dynamics of Vacancy-Induced Nanofilamentary Switching Network in Donor Doped $\mathrm{SrTiO}_{3-\mathrm{x}}$ Memristors. Nanotechnology 2016, 27 (50), 505210.

26. Ahmed, T.; Walia, S.; Mayes, E. L. H.; Ramanathan, R.; Guagliardo, P.; Bansal, V.; Bhaskaran, M.; Yang, J. J.; Sriram, S., Data Related to the Nanoscale Structural and Compositional Evolution in Resistance Change Memories. Data Brief 2018, 21, 18-24. 27. Valov, I.; Linn, E.; Tappertzhofen, S.; Schmelzer, S.; van den Hurk, J.; Lentz, F.; Waser, R., Nanobatteries in Redox-Based Resistive Switches Require Extension of Memristor Theory. Nat Commun 2013, 4, 1771.

28. Messerschmitt, F.; Kubicek, M.; Rupp, J. L. M., How Does Moisture Affect the Physical Property of Memristance for Anionic-Electronic Resistive Switching Memories? Advanced Functional Materials 2015, 25 (32), 5117-5125.

29. Valov, I.; Tsuruoka, T., Effects of Moisture and Redox Reactions in VCM And ECM Resistive Switching Memories. Journal of Physics D: Applied Physics 2018, 51 (41).

30. Liu, K.; Qin, L.; Zhang, X.; Zhu, J.; Sun, X.; Yang, K.; Cai, Y.; Yang, Y.; Huang, R., Interfacial Redox Processes in Memristive Devices Based on Valence Change and Electrochemical Metallization. Faraday Discuss 2019, 213 (0), 41-52.

31. Ahmed, T.; Walia, S.; Mayes, E. L. H.; Ramanathan, R.; Guagliardo, P.; Bansal, V.; Bhaskaran, M.; Yang, J. J.; Sriram, S., Inducing Tunable Switching Behavior in A Single Memristor. Applied Materials Today 2018, 11, 280-290.

32. Yeom, S. W.; Shin, S. C.; Kim, T. Y.; Ha, H. J.; Lee, Y. H.; Shim, J. W.; Ju, B. K., Transparent Resistive Switching Memory Using Aluminum Oxide on A Flexible Substrate. Nanotechnology 2016, 27 (7), 07LT01. 
33. Yang, Y.; Sheridan, P.; Lu, W., Complementary Resistive Switching in Tantalum Oxide-Based Resistive Memory Devices. Applied Physics Letters 2012, 100 (20), 203112. 34. Al-Hamadany, R.; Goss, J. P.; Briddon, P. R.; Mojarad, S. A.; O'Neill, A. G.; Rayson, M. J., Impact of Tensile Strain on the Oxygen Vacancy Migration in $\mathrm{SrTiO}_{3}$ : Density Functional Theory Calculations. Journal of Applied Physics 2013, 113 (22), 224108.

35. Al-Hamadany, R.; Goss, J. P.; Briddon, P. R.; Mojarad, S. A.; Al-Hadidi, M.; O'Neill, A. G.; Rayson, M. J., Oxygen Vacancy Migration In Compressively Strained $\mathrm{SrTiO}_{3}$. Journal of Applied Physics 2013, 113 (2), 024108.

36. Zhao, K. L.; Chen, D.; Li, D. X., First Principles Study of Interface Structure And Electronic Property of $\mathrm{Au} / \mathrm{SrTiO}_{3}(001)$. Computational Materials Science 2010, 50 (1), 98 104.

37. Mrovec, M.; Albina, J. M.; Meyer, B.; Elsässer, C., Schottky Barriers at TransitionMetal/SrTiO 3 (001)Interfaces. Physical Review B 2009, 79, 245121, 1-18.

38. Cai, H. L.; Wu, X. S.; Gao, J., Effect of Oxygen Content on Structural and Transport Properties in $\mathrm{SrTiO}_{3-\mathrm{x}}$ Thin Flms. Chemical Physics Letters 2009, 467 (4-6), 313-317. 39. Kim, S.; Abbas, Y.; Jeon, Y. R.; Sokolov, A. S.; Ku, B.; Choi, C., Engineering Synaptic Characteristics of $\mathrm{TaO}_{\mathrm{x}} / \mathrm{HfO}_{2} \mathrm{Bi}$-Layered Resistive Switching Device. Nanotechnology 2018, 29 (41), 415204. 


\section{Supporting Information}

for

\section{Differential work-function enabled bifunctional switching in strontium titanate flexible resistive memories}

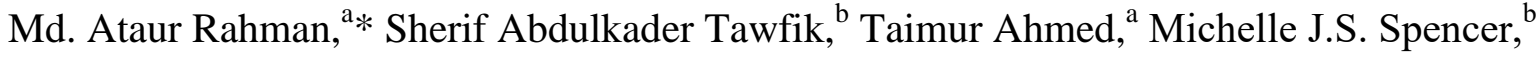
Sumeet Walia, ${ }^{a}$ Sharath Sriram, ${ }^{a}$ and Madhu Bhaskaran ${ }^{\mathrm{a} *}$

${ }^{a}$ Functional Materials and Microsystems Research Group and the Micro Nano Research Facility, RMIT University, Melbourne, Victoria 3001, Australia

${ }^{\mathrm{b}}$ School of Science, RMIT University, GPO Box 2476 Melbourne, Victoria 3001 Australia

*Corresponding authors email: mdataur.rahman@ student.rmit.edu.au, madhu.bhaskaran@rmit.edu.au

\section{Details of the work function difference Calculations}

Calculations were performed using density functional theory (DFT) as implemented in the Vienna $\mathrm{Ab}$ initio Simulation Package (VASP), ${ }^{\mathrm{S} 1}$ in which the ion-electron interaction is defined using the projector augmented wave (PAW) method. ${ }^{\mathrm{S} 2}$ A generalised gradient approximation (GGA) in the Perdew-Burke-Ernzerhof $(\mathrm{PBE})^{\mathrm{S} 3}$ form was used. We used a plane-wave energy cut-off of $500 \mathrm{eV}$, and a k-point mesh of $5 \times 5 \times 1$. For the energy minimization, we used a tolerance of $10-5 \mathrm{eV}$, and for the geometric optimization a force tolerance of $0.03 \mathrm{eV} / \AA$ was used. The work function is calculated using the formula $V-E_{F}$, where $\mathrm{V}$ is the vacuum potential which is maximum of the plane-averaged potential and $E_{F}$ is the Fermi level.

The four surfaces modelled are $\mathrm{Ti}(001), \mathrm{Au}(111), \operatorname{Pt}(111)$, and $\mathrm{Pd}(111)$. The unit cell of each of these surfaces is composed of a slab with a thickness of 4 unit cells. In the geometry optimization, the positions of the atoms in the lower two unit cells were frozen while the rest were left free to move. 


\section{Effect of device size on the switching behaviour}

We tested the behaviour of three devices for different sizes $40 \times 40 \mu \mathrm{m}^{2}, 20 \times 20 \mu \mathrm{m}^{2}$, $10 \times 10 \mu \mathrm{m}^{2}$ as shown in the Figure $\mathrm{S} 1$. We observed similar behaviour as $60 \times 60 \mu \mathrm{m}^{2}$. We can see slight variation from size to size, but overall there was negligible deviation with respect to switching type, switching direction, and switching voltages. It is well established that nanofilament is mainly responsible for switching the VCM devices. Oxygen vacancies are accountable for forming the nanofilament. The filamentary conduction of STO based memristor is discussed elaborately in our earlier report. ${ }^{S 4-7}$ If the size of the device is scaled down, the device operates stably due to filamentary switching.
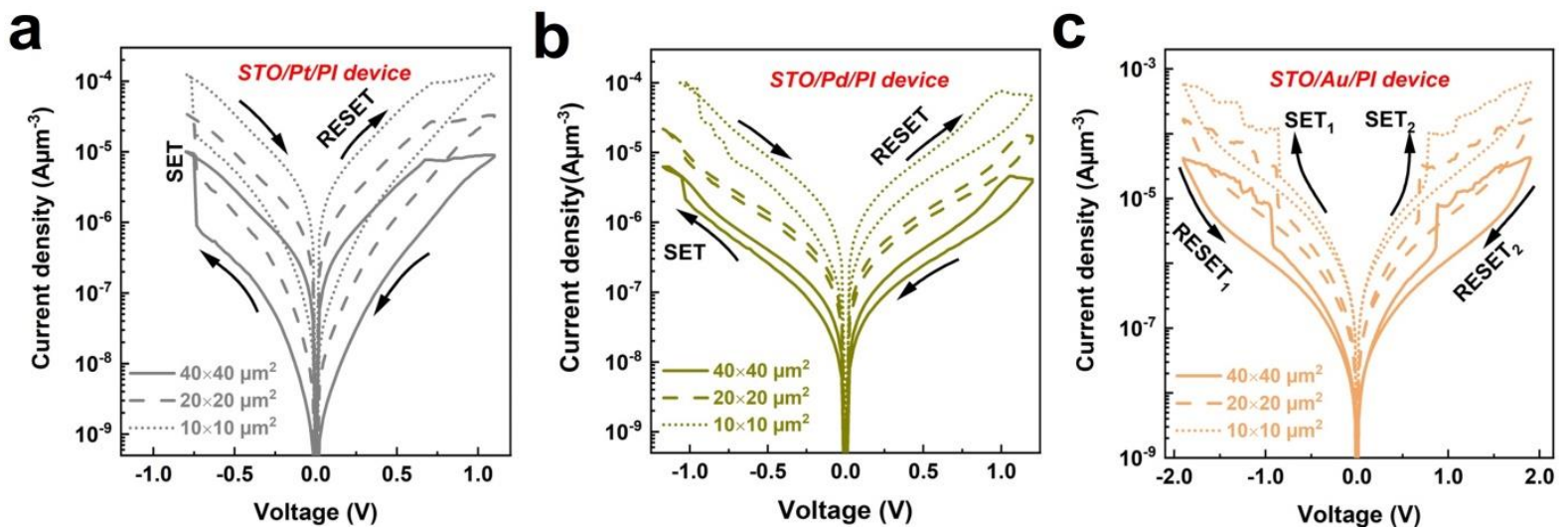

Figure S1. (a) Size dependent behaviour of STO/Pt/PI device (b) Size dependent behaviour of STO/Pd/PI device and (c) Size dependent behaviour of STO/Au/PI device.

\section{Test set up for moisture effect}

To understand the effect of moisture, we carried out further experiments on our devices under various relative humidity ranging from $10 \%$ to $90 \%$. In order to achieve that, a humidity controller RH 95 was connected to the LTS 420 stage (Linkam Scientific). The RH 95 is equipped with a specially designed automatic recycling desiccant system to dry the ambient air. To monitor the air supplied into the chamber, a sensor has also been placed inside the chamber to create a feedback loop to the controller as depicted in Figure S2. 


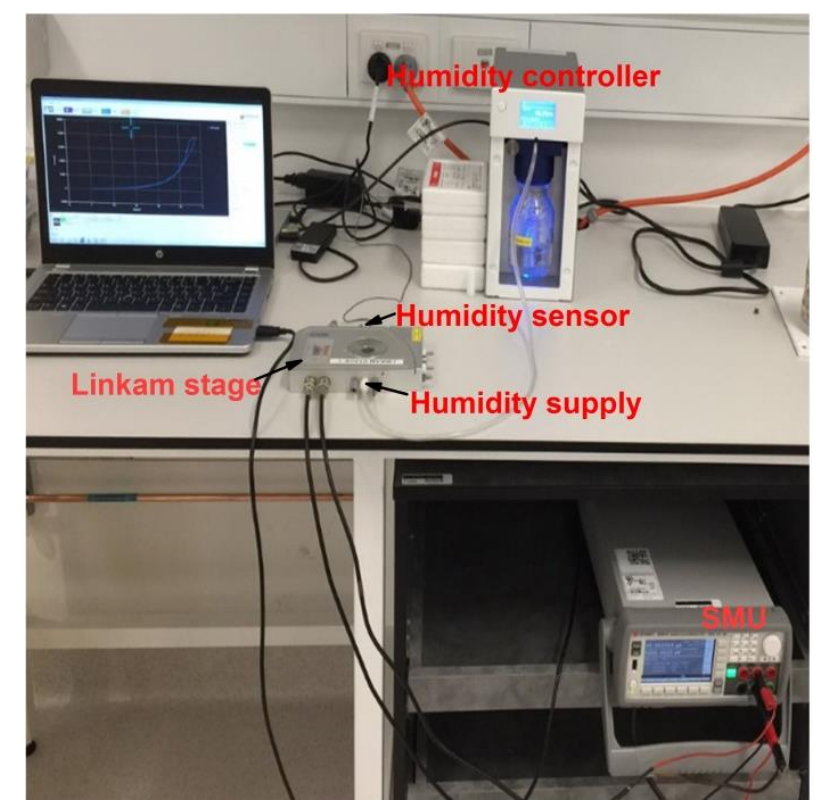

Figure S2. Testing setup for moisture effect.

\section{Bipolar switching Behaviour of STO/Pt/PI}

The repeatable and well-defined BP switching behaviours of our STO/Pt/PI stack for 50 cycles are represented in Figure $\mathrm{S} 3 \mathrm{a}$ on a log-scale after electroforming. After electroforming, the SET (low resistive state LRS) and RESET (high Resistive state-HRS) are achieved by applying DC voltage sweeps of opposite polarity. ${ }^{57}$ The cell turns $\mathrm{ON}$ by applying a negative voltage of $-0.85 \mathrm{~V}$ on the $\mathrm{STO} / \mathrm{Pt}$ interface, ${ }^{\mathrm{S} 8}$ turns $\mathrm{OFF}$ upon applying a positive voltage bias of $+1.15 \mathrm{~V}$. Figure $\mathrm{S} 3 \mathrm{~b}$ shows the stable retention characteristics for $10^{3} \mathrm{~s}$ with an average switching ratio (HRS/LRS) of $\sim 14$ at a constant read voltage $\left(V_{\text {READ }}\right)$ of $85 \mathrm{mV}$ which suggests the capability and stability of the bi-level storage of our devices. The endurance measurement in this work is carried out using a train of short pulses with duration of $1 \mathrm{~ms}$ for WRITE/ERASE/READ devices as depicted in Figure S3c (shows representative pulse scheme for two cycles). For each cycle, a negative SET pulse ( $\left.V_{\mathrm{SET}}\right)$ with amplitude of 1.0 V sets the devices to LRS followed by a READ pulse ( $\left.V_{\text {READ }}\right)$ pulse with amplitude of $85 \mathrm{mV}$, while a positive pulse $V_{\text {RESET }}$ of $1.15 \mathrm{~V}$ resets the devices to HRS trailed by a $V_{\text {READ }}$ pulse of same magnitude. The applied $V_{\mathrm{SET}}-V_{\mathrm{READ}}-V_{\mathrm{RESET}}-V_{\mathrm{READ}}$ voltage sequence cycles the device through the $\mathrm{ON}-\mathrm{LRS}-\mathrm{ON}-\mathrm{HRS}$ sequence. The endurance cycle characteristics are evaluated for 1,000 cycles demonstrating stable bipolar switching behaviour as shown in Figure S3d. This behaviour indicates that the bottom electrode, Pt with higher work function compared to that of Au coupled with an asymmetric applied bias $(-1.00 \mathrm{~V}$ to $+1.15 \mathrm{~V})$ results in BP switching. 

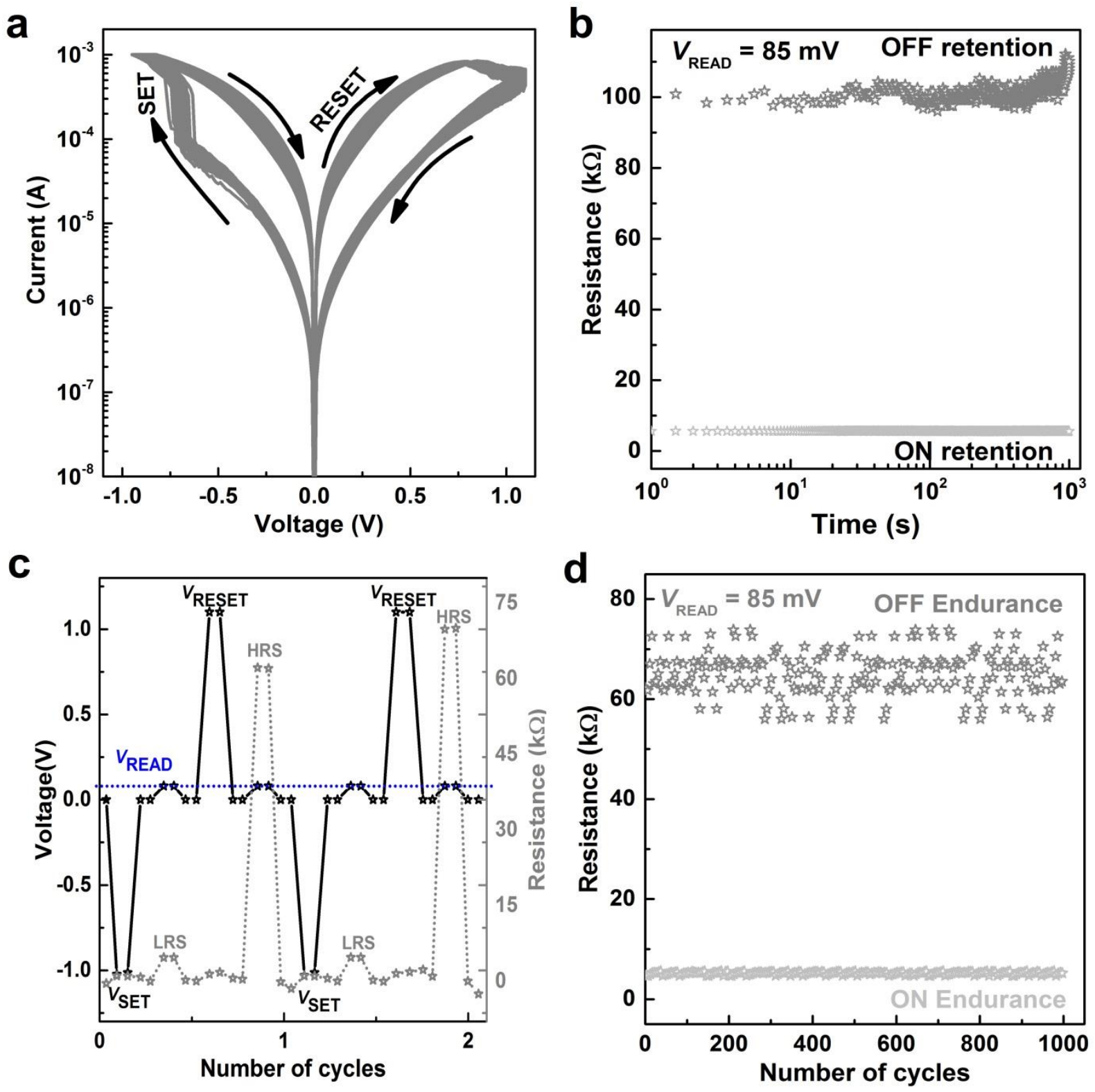

Figure S3. Polarity-dependent BP switching behaviour of STO/Pt/PI MIM devices. (a) Quasistatic bipolar switching for 50 cycles indicating stable memory behaviour. The asymmetric device is SET at $-0.75 \mathrm{~V}$ and RESET at $+1.15 \mathrm{~V}$ (b) Illustrates the data retention time for $10^{3} \mathrm{~s}$ at a constant $V_{\text {READ }}$ of $85 \mathrm{mV}$. (c) Pulse mode operation for two consecutive cycles with the pulse width of $1 \mathrm{~ms}$. Each cycle comprised of $V_{\mathrm{SET}}, V_{\mathrm{RESET}}$ and $V_{\mathrm{READ}}$ turns the device to the ON state, LRS state and HRS state. (d) Endurance for $10^{3}$ cycles at a read voltage of $85 \mathrm{mV}$

\section{Complementary switching behaviour of $S T O / A u / P I$}

Figure S4a represents the symmetric CS behaviour of our STO/Au/PI observed for 50 continuous switching cycles in $\log$ scale. The CS behaviour occurs due to the alternate formation and disruption of conductive filament in the surrounding area of the Schottky and ohmic interfaces owing to the polarity of biasing voltage. ${ }^{\mathrm{S} 9}$ The growth of the filament at an 
interface results in local low resistive state (LLRS) and rupture of the filament leads to local high resistive state (LHRS). For instance, the application of negative bias at Schottky (STO/Au) interface causes the device switch to its $\mathrm{SET}_{1}$ state with fully grown conductive filament. Further, increasing the negative bias forces the device to the RESET $_{1}$ state where $V_{\mathrm{o}} \mathrm{S}$ are heavily concentrated towards the Schottky interface (LLRS) generating a partial depletion region at ohmic (Ti/STO) interface (LHRS). As a result, the RESET $_{1}$ state offers higher resistivity than $\mathrm{SET}_{1}$. On the contrary, application of a reverse bias reverts $V_{\mathrm{o}} \mathrm{s}$ towards ohmic interface resulting in a sequential $\mathrm{SET}_{2}$ state before transitioning to the RESET 2 due to higher applied voltage. ${ }^{\text {S10 }}$

Contrary to BP ReRAM devices, a CS device only offers a $I-V$ based readout between $\pm 1.0 \mathrm{~V}$ and $\pm 1.9 \mathrm{~V}$ to retrieve the stored information from the CS cell, since the two high resistive storing states are not distinguishable at low voltage. ${ }^{\mathrm{S} 9,11}$ Consequently, the devices are read at $1.3 \mathrm{~V}$ to enable the SET state $\left(\mathrm{SET}_{1}\right.$ or $\mathrm{SET}_{2}$ ). The retention and endurance characteristics are acquired at $1.3 \mathrm{~V}$ for $10^{3} \mathrm{~s}$ and $10^{3}$ cycles, respectively. The distinct $\mathrm{OFF}$ and $\mathrm{ON}$ retention without any degradation elucidates the capability and stability of the switching performance of our devices as depicted in Figure S4b.

Similar to BP characterisations, the endurance measurement is accomplished using a series of pulses in a sequence of $V_{-}(-2.0 \mathrm{~V})-V_{\operatorname{READ}}(1.3 \mathrm{~V})-V_{+}(+2.1 \mathrm{~V})-V_{\mathrm{READ}}(1.3 \mathrm{~V})$ with a pulse width of $1 \mathrm{~ms}$ as represented in Figure S4c. A complete cycle consists of $V_{-}-V_{\mathrm{READ}}-V_{+}-$ $V_{\mathrm{READ}}$ switches the device in a sequence of $\mathrm{SET}_{1}-\mathrm{RESET}_{1}-\mathrm{SET}_{2}-\mathrm{RESET}_{2}$ states. The RESET $_{1}$ current is obtained after setting the device on $V_{-}$for $V_{\text {READ }}$ whereas RESET 2 current corresponds to the device set on $V_{+}$for same $V_{\mathrm{READ}}$. RESET ${ }_{1}$ and $\mathrm{RESET}_{2}$ therefore directly correspond to the ON and OFF endurances, respectively. The endurance test conducted for 1000 cycles without any substantial degradation infer the stable CS characteristic even at a relatively higher $V_{\mathrm{READ}}$ of $1.3 \mathrm{~V}$ as shown in Figure $\mathrm{S} 4 \mathrm{~d}$. From the retention test as well as the cyclic endurance retention, the switching ratio (ON/OFF) of $\sim 6$ is observed. 

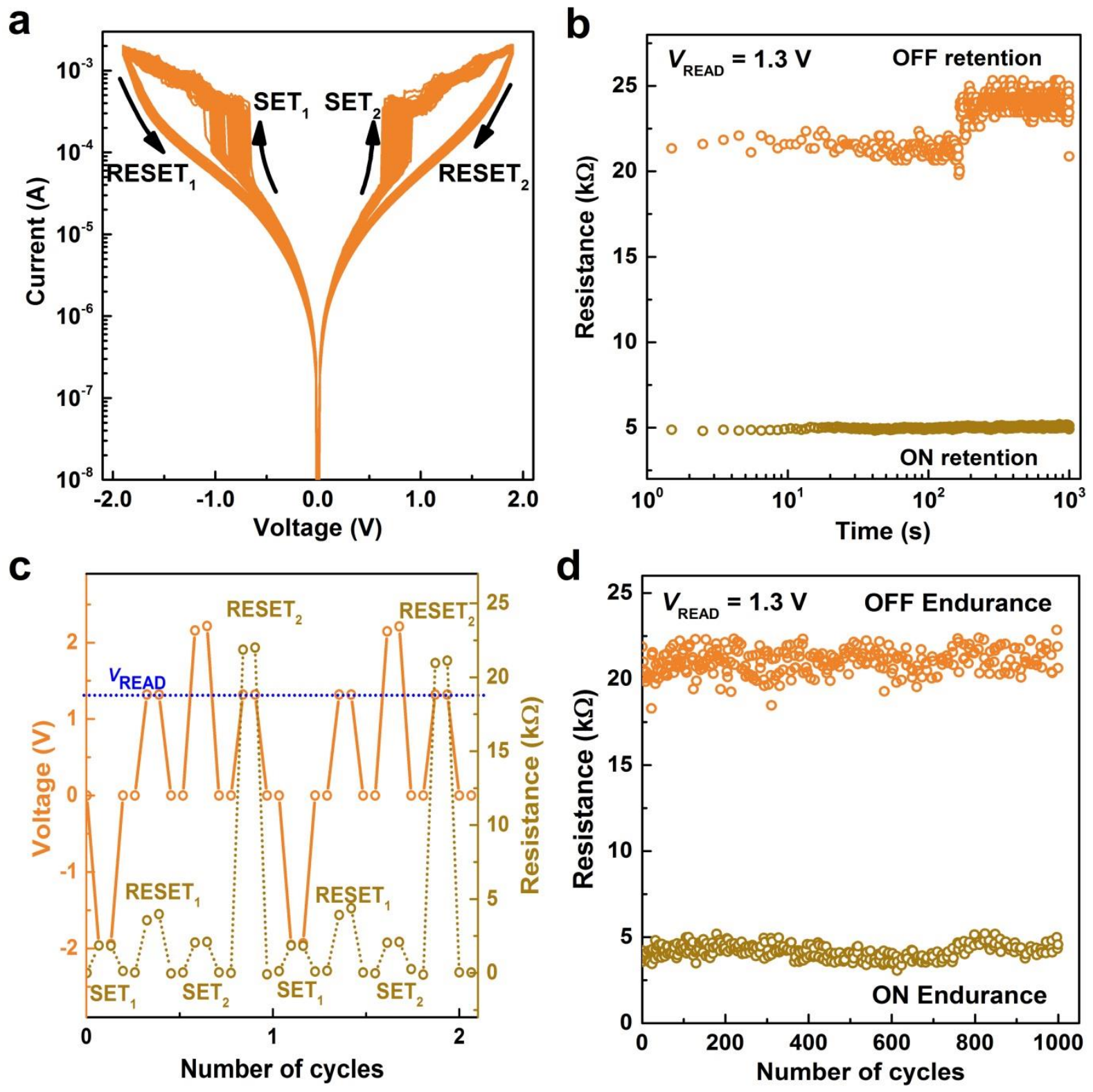

Figure S4. CS memory performance of STO/Au/PI MIM devices. (a) Complementary switching for 50 cycles where the arrow marks direct the switching. The ON state is represented as $\mathrm{SET}_{1}$ and $\mathrm{SET}_{2}$ for positive and negative half cycles, respectively. (b) Stable retention test for $1000 \mathrm{~s}$ with a $V_{\text {READ }}$ of $1.3 \mathrm{~V}$ (c) Endurance test procedure using a series of pulses for two representative cycles. The applied $V_{-}-V_{\mathrm{READ}}-V_{+}-V_{\mathrm{READ}}$ voltage sequence switches the device to $\mathrm{SET}_{1}-\mathrm{RESET}_{1}-\mathrm{SET}_{2}-\mathrm{RESET}_{2}$ sequence. (d) Endurance for 1,000 cycles at read voltage of $1.3 \mathrm{~V}$. 


\section{Switching behaviour of Pd/Ti/STO/Pt/Ti and Pd/Ti/STO/Au/Ti}

Figure S5 depicts the switching characteristic of the devices where the top Pt and Au were replaced by $\mathrm{Pd}$ to determine the role of the top metal electrode in the switching mechanism. Bottom electrodes were retained the same for comparison. The bipolar switching and complementary switching from devices with Pd top electrodes confirm that the bottom electrodes determine switching, with the top inert electrodes primary role being protecting of the $10 \mathrm{~nm}$ Ti layer above STO from external oxidation.
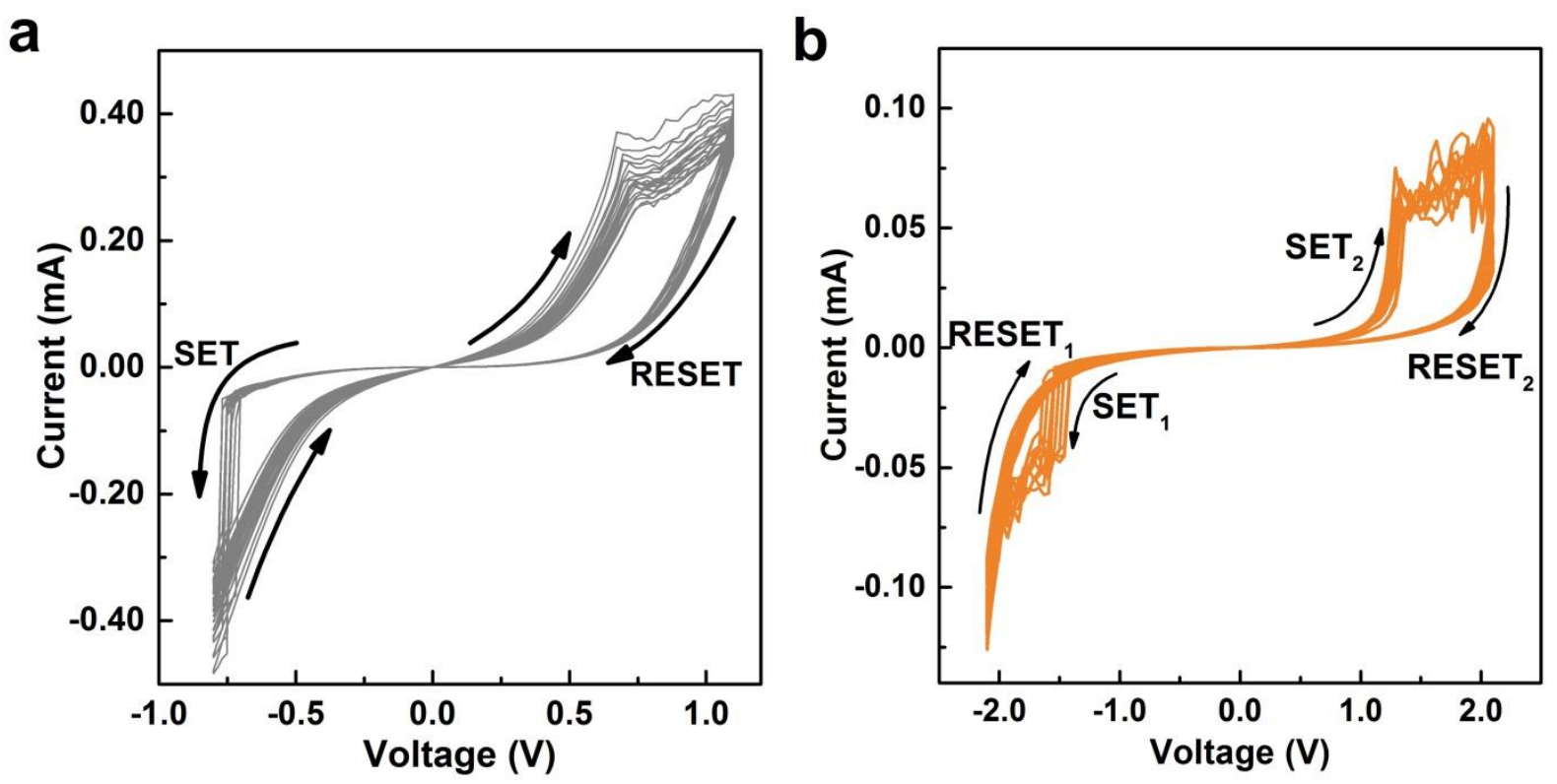

Figure S5. Representative (a) bipolar switching in $\mathrm{Pd} / \mathrm{Ti} / \mathrm{STO} / \mathrm{Pt} / \mathrm{Ti} / \mathrm{PI}$ and (b) complementary switching in $\mathrm{Pd} / \mathrm{Ti} / \mathrm{STO} / \mathrm{Au} / \mathrm{Cr} / \mathrm{PI}$. 


\section{References}

S1. Kresse, G.; Furthmüller, J., Efficient Iterative Schemes for Ab Initio Total-Energy Calculations using A Plane-Wave Basis Set. Physical Review B 1996, 54 (16), 11169-11186. S2. Blöchl, P. E., Projector Augmented-Wave Method. Physical Review B 1994, 50 (24), 17953-17979.

S3. Perdew, J. P.; Burke, K.; Ernzerhof, M., Generalized Gradient Approximation Made Simple. Physical Review Letters 1996, 77 (18), 3865-3868.

S4. Rahman, M. A.; Ahmed, T.; Walia, S.; Sriram, S.; Bhaskaran, M., Oxygen-Deficient Strontium Titanate Based Stretchable Resistive Memories. Applied Materials Today 2018, 13, 126-134.

S5. Rahman, F.; Ahmed, T.; Walia, S.; Mayes, E.; Sriram, S.; Bhaskaran, M.; Balendhran, S., Reversible Resistive Switching Behaviour in CVD Grown, Large Area Moox. Nanoscale 2018, 10 (42), 19711-19719.

S6. Nili, H.; Ahmed, T.; Walia, S.; Ramanathan, R.; Kandjani, A. E.; Rubanov, S.; Kim, J.; Kavehei, O.; Bansal, V.; Bhaskaran, M.; Sriram, S., Microstructure and Dynamics af Vacancy-Induced Nanofilamentary Switching Network in Donor Doped $\mathrm{SrTiO}_{3-\mathrm{X}}$ Memristors. Nanotechnology 2016, 27 (50), 505210.

S7. Nili, H.; Walia, S.; Kandjani, A. E.; Ramanathan, R.; Gutruf, P.; Ahmed, T.; Balendhran, S.; Bansal, V.; Strukov, D. B.; Kavehei, O.; Bhaskaran, M.; Sriram, S., DonorInduced Performance Tuning of Amorphous $\mathrm{SrTiO}_{3}$ memristive Nanodevices: Multistate Resistive Switching And Mechanical Tunability. Advanced Functional Materials 2015, 25 (21), 3172-3182.

S8. Waser, R.; Dittmann, R.; Staikov, G.; Szot, K., Redox-Based Resistive Switching Memories - Nanoionic Mechanisms, Prospects, and Challenges. Advanced Materials 2009, 21 (25-26), 2632-2663.

S9. $\quad$ Breuer, T.; Siemon, A.; Linn, E.; Menzel, S.; Waser, R.; Rana, V., A HfO2-Based Complementary Switching Crossbar Adder. Advanced Electronic Materials 2015, 1 (10), 1500138.

S10. Breuer, T.; Nielen, L.; Roesgen, B.; Waser, R.; Rana, V.; Linn, E., Realization of Minimum and Maximum Gate Function in Ta2O5-based Memristive Devices. Sci Rep 2016, 6, 23967.

S11. Ahmed, T.; Walia, S.; Mayes, E. L. H.; Ramanathan, R.; Guagliardo, P.; Bansal, V.; Bhaskaran, M.; Yang, J. J.; Sriram, S., Inducing Tunable Switching Behavior in A Single Memristor. Applied Materials Today 2018, 11, 280-290. 